\title{
Decision support model with Pythagorean fuzzy preference relations and its application in financial early warnings
}

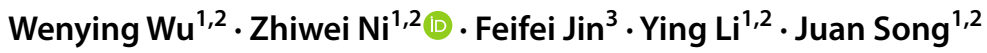

Received: 10 July 2020 / Accepted: 26 April 2021 / Published online: 27 May 2021

(c) The Author(s) 2021

\begin{abstract}
Pythagorean fuzzy sets (PFSs) retain the advantages of intuitionistic fuzzy sets (IFSs), while PFSs portray 1.57 times more information than IFSs. In addition, Pythagorean fuzzy preference relations (PFPRs), as a generalization of intuitionistic fuzzy preference relations (IFPRs), are more flexible and applicable. The objective of this paper is to propose a novel decision support model for solving group decision-making problems in a Pythagorean fuzzy environment. First, we define the concepts of ordered consistency and multiplicative consistency for PFPRs. Then, aiming at the group decision-making problem of multiple PFPRs, a consistency improving model is constructed to improve the consistency of group preference relations. Later, a consensus reaching model is developed to reach the degree of group consensus. Furthermore, a decision support model with PFPRs is established to derive the normalized weights and output the final result. Holding these features, this paper builds a decision support model with PFPRs based on multiplicative consistency and consensus. Finally, the described method is validated by an example of financial risk management, and it is concluded that the solvency of a company is an important indicator that affects the financial early warning system.
\end{abstract}

Keywords Pythagorean fuzzy preference relation $\cdot$ Multiplicative consistency $\cdot$ Consensus $\cdot$ Decision support model · Financial crisis and risk management

Zhiwei Ni

zhiwein@163.com

Wenying $\mathrm{Wu}$

wuwenying0318@163.com

Feifei Jin

shexian19880129@163.com

Ying Li

hgdly@126.com

Juan Song

songjuan134@163.com

1 School of Management, Hefei University of Technology, Hefei 230009, Anhui, China

2 Key Laboratory of Process Optimization and Intelligent Decision-Making, Ministry of Education, Hefei 230009, Anhui, China

3 School of Business, Anhui University, Hefei 230601, Anhui, China

\section{Introduction}

Decision-making implies that there is a range of choices. In this case, decision-makers (DMs) rank these choices from best to worst, and choose a goal that meets the expectations of DMs. In group decision-making (GDM) problems, DMs usually need to give a precise preference for a group of options using pairwise comparisons to express their preferred information and construct a preference relation judgment matrix [1-3]. Obviously, there are many methods to support decision-making [4-6]. Nevertheless, in many GDM problems, DMs may be challenging to depict their preference with a precise number. One reason is that DMs may not have an accurate or sufficient knowledge level of the problem, and the other is that DMs cannot clearly distinguish the extent to which one is superior to others [7, 8]. In these cases, DMs may prefer to compare the imprecise judgment information in a matrix in pairs. To describe this vagueness and indeterminacy, researchers have introduced fuzzy sets (FSs) [9] theory to different preference relations (PRs). Then, fuzzy preference relations (FPRs) [10] and interval fuzzy preference relations (IFPRs) [11] emerged. Compared 
with traditional PRs, FPRs can provide reasonable feedback on the preferences given by DMs via a membership function [12-14]. With the growing complexity of the decision-making environment, there will be recognitions of affirmation, hesitation, or negation in decision-making. Therefore, IFPRs can feedback assessment intuitively with membership, nonmembership, and hesitation [15-20].

However, the domain inscribed by the IFSs [21, 22] can be visualized as a triangular domain using a two-dimensional coordinate axis. Yager et al. [23, 24] proposed PFSs, which are a further extension of IFSs. PFSs not only possess a membership function and non-membership function, but also extend the area composed of two functions to 1/4 circles. While maintaining the characteristics of IFSs, PFSs have a wider range. Therefore, PFSs are more prominent in handling decision-making problems. Currently, the research on PFSs mainly involves aggregation operators [25-27], similarity measures and distance measures [28-30], and extensions of classical methods [31,32]. To give a clear perspective on the different concepts, tools, and trends associated with their extension, Peng and Selvachandran [33] provided an overview of PFSs.

Given the development of PFSs, PFSs were introduced to PRs, the concept of Pythagorean fuzzy reference relations (PFPRs) $[34,35]$ was proposed, and the GDM problem under PFPRs was studied.

The core of the decision-making problem based on PRs includes how to define the consistency of PRs and how to obtain the weight vector of PRs. Chu et al. [18] constructed a GDM framework with IFPRs based on additive consistency and the group consensus. Jin et al. [19] designed two methods to obtain the normalized priority weights from IFPRs. To represent preferences under uncertain weights, Lin and Wang [20] minimized extreme deviation from the relevant consistent IFPRs. Zhang [36] proposed a new approach index of Pythagorean fuzzy numbers (PFNs) and introduced a PFN ranking method based on the approach index. Additionally, the concept of the interval-valued Pythagorean fuzzy set (IVPFS) parallel to the interval intuitionistic fuzzy set was proposed [36]. In view of the imprecise modeling ability of PFS for human perception in multi-criteria decision-making, Zhang et al. [36] introduced the classic preference ranking organization method of enrichment evaluations (PROMETHEE) in the Pythagorean fuzzy environment. In Zhang et al.'s method [37], the weights and preferences are considered synthetically, which increase the range of the decision-maker's choice. Based on a new multiplicative consistency definition of interval fuzzy preference relations, Zhang [38] constructs a goal programming model.

However, when using additive consistency to estimate incomplete elements in FPRs, it is easy to exclude partial incomplete elements from $[0,1]$. Thus, the final decision result may be unreliable. In some cases, additive consistency is not suitable for fuzzy preference relations. In contrast, the multiplicative consistency in FPRs can effectively compensate for this deficiency. Furthermore, Jin et al. [19] discussed multiplicatively consistent IFPRs and constructed a group decision model for solving practical problems. However, we know that the area of an intuitionistic fuzzy set (IFS) can be regarded as a triangle while the area of a Pythagorean fuzzy set (PFS) is a quarter circle. In short, the spatial membership of a PFS is greater than that of an IFS, which is equivalent to an intuitionistic fuzzy number (IFN) being a PFN. Otherwise, it does not hold [22, 23]. Therefore, PFPRs can solve the problem that IFPRs are incompetent. Yang et al. [35] defined additive consistent PFPRs and Pythagorean fuzzy weighted quadratic (PFWQ) operators to construct group decision methods. In the GDM process, due to the complexity of the decision-making problem and the limitation of the evaluator's knowledge or background, not all PRs provided by each evaluator can meet the condition of additive consistency. Therefore, an optimization model with the minimum deviation between the given PFPR and the constructed multiplicatively consistent PFPR is established, and the objective optimization model is solved to obtain the fuzzy weight vector of the preference relation. It is worth affirming that Mandal and Ranadive [34] introduced PFPRs. However, the qualified consensus relation cannot be obtained through the given group collective PR [34]. He et al. [39] gave the multiplicative PFPR without considering the consensus among multiple DMs. Hence, it is only suitable for individual decision-making. In summary, how to generate a decision support model based on acceptably multiplicatively consistent PFPR and consensus is of great significance for GDM problems. Although it is worthwhile to discuss the multiplicative consistency under PFPRs, there are few related achievements. It is necessary to discuss the decision support model from the perspective of multiplicative consistency and consensus.

Taking the essential advantages of the Pythagorean fuzzy environment and multiplicative consistency to describe preference values, the main contributions of this paper are as follows.

1. We define the order consistency and multiplicative consistency with PFPRs.

2. We present three algorithms for ordered consistency, multiplicative consistency, and group consensus with PFPRs, respectively.

3. We demonstrate the proposed decision support model with an application in financial early warning to indicate its practicability and effectiveness.

The remainder of this paper is organized as follows. In "Preliminaries", some basic concepts, including FPRs, PFSs, and PFPRs, are briefly reviewed. "Method for deriving 
priority weight vectors with PFPRs" defines the multiplicative consistency and properties of PFPRs. "Multiplicative consistency improving algorithm" introduces consensus reaching with multiplicatively consistent PFPRs. Then, "Algorithm for group decision-making with PFPRs" develops a model for group decision-making with PFPRs. "Numerical example and comparison analysis" presents one illustrative example of financial early warning, and comparative analyses are implemented simultaneously on the proposed approach and other existing methods. Finally, conclusions are summarized in "Conclusions".

\section{Preliminaries}

To illustrate this further, we introduce some basic concepts.

\section{Fuzzy preference relations}

Definition 1 [10] Assume that $X=\left\{x_{1}, x_{2}, \ldots, x_{n}\right\}$ is a finite set of alternatives. A fuzzy preference relation $R$ on $X$ is defined by a reciprocal matrix $R=\left(r_{i j}\right)_{n \times n}$ with the circumstance that

$0 \leq r_{i j} \leq 1, r_{i j}+r_{j i}=1, r_{i i}=0.5, \quad i, j=1,2, \ldots, n$,

where $r_{i j}$ indicates the degree of preference of alternative $x_{i}$ relative to $x_{j}$.

If $r_{i j}=0.5$, there is no difference between alternative $x_{i}$ and alternative $x_{j}$. If $r_{i j}>0.5$, alternative $x_{i}$ is superior to alternative $x_{j}$. If $r_{i j}=1$, alternative $x_{i}$ is completely superior to alternative $x_{j}$.

\section{Intuitionistic fuzzy preference relations}

Definition 2 [15] Assume that $X=\left\{x_{1}, x_{2}, \ldots, x_{n}\right\}$ is a finite set. The intuitionistic fuzzy preference relation is denoted as

$I=\left(\tilde{p}_{i j}\right)_{n \times n}=\left(\left\langle\rho_{i j}, \sigma_{i j}\right\rangle\right)_{n \times n}$,

where $\rho_{I}\left(x_{i}\right)$ and $\sigma_{I}\left(x_{i}\right)$ represent the degree of membership and degree of non-membership of the elements $x_{i} \in X$ to the set $I$, respectively. Let $\alpha=(\rho, \sigma)=\left(\rho_{I}\left(x_{i}\right), \sigma_{I}\left(x_{i}\right)\right)$ be IFN where

$\rho, \sigma \in[0,1], 0 \leq \rho_{i j}+\sigma_{i j} \leq 1, \rho_{i j}=\sigma_{j i}, \sigma_{i j}=\rho_{j i}, \rho_{i j}=\sigma_{j i}=0.5$,

and $i, j \in N$.

Definition 3 [15] Let $\alpha_{i}=\left(\rho_{i}, \sigma_{i}\right), i=1,2$ be double IFNs that satisfy the following sequential relations:

1. If $\Delta\left(\alpha_{1}\right)<\Delta\left(\alpha_{2}\right)$, then $\alpha_{1}<\alpha_{2}$.
2. If $\Delta\left(\alpha_{1}\right)=\Delta\left(\alpha_{2}\right)$, and $\varphi\left(\alpha_{1}\right)<\varphi\left(\alpha_{2}\right)$, then $\alpha_{1}<\alpha_{2}$.

3. If $\Delta\left(\alpha_{1}\right)=\Delta\left(\alpha_{2}\right)$, and $\varphi\left(\alpha_{1}\right)=\varphi\left(\alpha_{2}\right)$, then $\alpha_{1}=\alpha_{2}$.

Here, the score function $\Delta\left(\alpha_{i}\right)=\rho_{i}-\sigma_{i}$, and the accuracy function $\varphi\left(\alpha_{i}\right)=\rho_{i}+\sigma_{i}$.

\section{Pythagorean fuzzy set}

Definition $4[23,24]$ Assume that $X=\left\{x_{1}, x_{2}, \ldots, x_{n}\right\}$ is a finite set. The Pythagorean fuzzy set is denoted as

$A=\left\{\left\langle x_{i}, \rho_{A}\left(x_{i}\right), \sigma_{A}\left(x_{i}\right)\right\rangle \mid x_{i} \in X\right\}$,

where $\rho_{A}\left(x_{i}\right)$ and $\sigma_{A}\left(x_{i}\right)$ represent the degree of membership and degree of non-membership of the elements $x_{i} \in X$ to the set $A$, respectively. Let $\alpha=(\rho, \sigma)=\left(\rho_{A}\left(x_{i}\right), \sigma_{A}\left(x_{i}\right)\right)$ be a PFN, where $\rho, \sigma \in[0,1], \rho^{2}+\sigma^{2} \leq 1$, and the indeterminate degree is $\pi_{A}=\sqrt{1-\rho^{2}-\sigma^{2}}$.

Definition 5 [40] Let $\alpha_{i}=\left(\rho_{i}, \sigma_{i}\right), i=1,2$ be PFNs. Then, we have the following:

1. If $s\left(\alpha_{1}\right)<s\left(\alpha_{2}\right)$, then $\alpha_{1}<\alpha_{2}$.

2. If $s\left(\alpha_{1}\right)=s\left(\alpha_{2}\right)$, and $h\left(\alpha_{1}\right)<h\left(\alpha_{2}\right)$, then $\alpha_{1}<\alpha_{2}$.

3. If $s\left(\alpha_{1}\right)=s\left(\alpha_{2}\right)$, and $h\left(\alpha_{1}\right)=h\left(\alpha_{2}\right)$, then $\alpha_{1}=\alpha_{2}$.

Here, the score function $s\left(\alpha_{i}\right)=\rho_{i}^{2}-\sigma_{i}^{2}$, and the accuracy function $h\left(\alpha_{i}\right)=\rho_{i}^{2}+\sigma_{i}^{2}$.

\section{Pythagorean fuzzy preference relations}

Definition $6[34,35]$ Let $\tilde{p}_{i j}(i, j \in N)$ be the PFNs and $P$ be the Pythagorean fuzzy preference relation defined on the alternative set $X$, which is characterized by

$$
P=\left(\tilde{p}_{i j}\right)_{n \times n}=\left(\left\langle\rho_{i j}, \sigma_{i j}\right\rangle\right)_{n \times n},
$$

with the condition that $\rho_{i j}=\sigma_{j i}, \sigma_{i j}=\rho_{j i}, \rho_{i i}=\sigma_{i i}=\sqrt{2} / 2$.

Generally, $\rho_{i j}$ represents the degree of preference of alternative $X_{i}$ relative to $X_{j}$, and $\sigma_{i j}$ represents the degree of preference of alternative $X_{j}$ relative to $X_{i}$. The indeterminate $\mathrm{d} \mathrm{e} \mathrm{g} \mathrm{r} \mathrm{e} \mathrm{e} \quad \tau_{i j}=\sqrt{1-\rho_{i j}^{2}-\sigma_{i j}^{2}} \quad$ a n d $\tau_{i i}=\sqrt{1-\rho_{i i}^{2}-\sigma_{i i}^{2}}=0, i \in N$.

\section{Method for deriving priority weight vectors with PFPRs}

\section{Multiplicative consistency and properties of PFPRs}

Definition 7 A PFPR $P=\left(\tilde{p}_{i j}\right)_{n \times n}, i, j \in N$, which satisfies 
$p_{\sigma(1), j} \geq p_{\sigma(2), j} \geq \cdots \geq p_{\sigma(n), j}$,

for all js, is called an order consistent PFPR.

Example 1 Let $X=\left\{x_{1}, x_{2}, \ldots, x_{n}\right\}$ be a set of alternatives. A PFPR $P=\left(\tilde{p}_{i j}\right)_{4 \times 4}, i, j \in N$ is as follows:

$P=\left[\begin{array}{cccc}(\sqrt{2} / 2, \sqrt{2} / 2) & (0.2,0.1) & (0.3,0.1) & (0.5,0.2) \\ (0.1,0.2) & (\sqrt{2} / 2, \sqrt{2} / 2) & (0.4,0.3) & (0.6,0.4) \\ (0.1,0.3) & (0.3,0.4) & (\sqrt{2} / 2, \sqrt{2} / 2) & (0.5,0.4) \\ (0.2,0.5) & (0.4,0.6) & (0.4,0.5) & (\sqrt{2} / 2, \sqrt{2} / 2)\end{array}\right]$.

From the above-mentioned PFPR $P$, it is apparent that $p_{1 j} \geq p_{2 j} \geq p_{3 j} \geq p_{4 j}, j \in\{1,2,3,4\}$, which denotes that alternative $x_{4}$ is inferior to $x_{3}$, alternative $x_{3}$ is inferior to $x_{2}$, and alternative $x_{2}$ is inferior to $x_{1}$. Therefore, according to the ordered consistency of PFPRs, the rank of the alternative sets is $x_{4}<x_{3}<x_{2}<x_{1}$.

Definition 8 Suppose that the PFPR $P=\left(\tilde{p}_{i j}\right)_{n \times n}, i, j \in N$ is regarded as multiplicatively consistent, which satisfies the multiplicative transitivity

$\rho_{i j}^{2} \cdot \rho_{j k}^{2} \cdot \rho_{k i}^{2}=\rho_{i k}^{2} \cdot \rho_{k j}^{2} \cdot \rho_{j i}^{2}, i, j, k=1,2, \ldots, n$.

For $\rho_{i j}=\sigma_{j i}, \sigma_{i j}=\rho_{j i}, i, j=1,2, \ldots, n$ in Definition 4, Eq. (6) is equivalent to the following:

$\sigma_{i j}^{2} \cdot \sigma_{j k}^{2} \cdot \sigma_{k i}^{2}=\sigma_{i k}^{2} \cdot \sigma_{k j}^{2} \cdot \sigma_{j i}^{2}, i, j, k=1,2, \ldots, n$.

Based on Definition 3.2, we introduce the following theorem.

Theorem 1 Assume that the PFPR $P=\left(\tilde{p}_{i j}\right)_{n \times n}=\left(\left\langle\rho_{i j}, \sigma_{i j}\right\rangle\right)_{n \times n}$ is multiplicative consistent, if and only if

$\mu\left(\tilde{p}_{i j}\right)=\mu\left(\tilde{p}_{i k}\right) \cdot \mu\left(\tilde{p}_{k j}\right), \quad i, j, k=1,2, \ldots, n$,

where $\mu\left(\tilde{p}_{i j}\right)=\rho_{i j}^{2} / \sigma_{i j}^{2}, i, j=1,2, \cdots, n$.

\section{Proof}

1. We first prove the necessity of Theorem 1 .

Obviously, by Definition 3.2, we know that $\rho_{i j}^{2} \cdot \rho_{j k}^{2} \cdot \rho_{k i}^{2}=\rho_{i k}^{2} \cdot \rho_{k j}^{2} \cdot \rho_{j i}^{2}, \quad i, j, k=1,2, \ldots, n$. Since $\rho_{i j}=\sigma_{j i}, \sigma_{i j}=\rho_{j i}, \quad i, j=1,2, \ldots, n$, then the expression of $\rho_{i j}^{2} \cdot \sigma_{k j}^{2} \cdot \sigma_{i k}^{2}=\rho_{i k}^{2} \cdot \rho_{k j}^{2} \cdot \sigma_{i j}^{2}, \quad i, j, k=1,2, \ldots, n$ can be obtained. Furthermore, we have the equation $\frac{\rho_{i j}^{2}}{\sigma_{i j}^{2}}=\frac{\rho_{i k}^{2}}{\sigma_{i k}^{2}} \cdot \frac{\rho_{k j}^{2}}{\sigma_{k j}^{2}}, \quad i, j, k=1,2, \ldots, n$.

Hence, from Eq. (9), it follows that $\mu\left(\tilde{p}_{i j}\right)=\mu\left(\tilde{p}_{i k}\right) \cdot \mu\left(\tilde{p}_{k j}\right), \quad i, j, k=1,2, \ldots, n$.

2. Here, we continue to prove the sufficiency of Theorem 1 .
Since $\rho_{i j}=\sigma_{j i}, \sigma_{i j}=\rho_{j i}, \quad i, j=1,2, \ldots, n \quad$ and $\mu\left(\tilde{p}_{i j}\right)=\frac{\rho_{i j}^{2}}{\sigma_{i j}^{2}}, \quad i, j=1,2, \ldots, n$, then we know

$\frac{\rho_{i j}^{2}}{\sigma_{i j}^{2}}=\frac{\rho_{i k}^{2}}{\sigma_{i k}^{2}} \cdot \frac{\rho_{k j}^{2}}{\sigma_{k j}^{2}}, \quad i, j, k=1,2, \ldots, n$. Considering the above Proof (1), one can obtain

$\rho_{i j}^{2} \cdot \rho_{j k}^{2} \cdot \rho_{k i}^{2}=\rho_{i k}^{2} \cdot \rho_{k j}^{2} \cdot \rho_{j i}^{2}, \quad i, j, k=1,2, \ldots, n$,

which indicates that $P=\left(\tilde{p}_{i j}\right)_{n \times n}=\left(\left\langle\rho_{i j}, \sigma_{i j}\right\rangle\right)_{n \times n}$ is multiplicative consistency.

Definition 9 Suppose that a Pythagorean fuzzy weight vector $w=\left\{w_{1}, w_{2}, \ldots, w_{n}\right\}^{T}$ of PFPR $P=\left(\tilde{p}_{i j}\right)_{n \times n}=\left(\left\langle\rho_{i j}, \sigma_{i j}\right\rangle\right)_{n \times n}$ is regarded to be standardized, which meets the following conditions:

$\sum_{j \neq i}^{n}\left(w_{j}^{\rho}\right)^{2} \leq\left(w_{i}^{\sigma}\right)^{2}$

$\sum_{j \neq i}^{n}\left(w_{j}^{\sigma}\right)^{2} \leq\left(w_{i}^{\rho}\right)^{2}+n-2, \quad i=1,2, \ldots, n$,

where $w_{i} \equiv\left(w_{i}^{\rho}, w_{i}^{\sigma}\right)$ is a PFV, a n d $0 \leq w_{i}^{\rho}, w_{i}^{\sigma} \leq 1,\left(w_{i}^{\rho}\right)^{2}+\left(w_{i}^{\sigma}\right)^{2} \leq 1$.

Based on the above-mentioned normalized Pythagorean fuzzy weight vector $w=\left\{w_{1}, w_{2}, \ldots, w_{n}\right\}^{T}$, a multiplicatively consistent PFPR $G_{p}=\left(\tilde{g}_{i j}\right)_{n \times n}$ is constructed.

Theorem 2 A PFPR $G_{p}=\left(\tilde{g}_{i j}\right)_{n \times n}$ is multiplicative consistent PFPR, and it is expressed as follows:

$\tilde{g}_{i j}= \begin{cases}(\sqrt{2} / 2, \sqrt{2} / 2), & i=j, \\ \left(\sqrt[4]{\left(w_{i}^{\rho}\right)^{2} \cdot\left(w_{j}^{\sigma}\right)^{2}}, \sqrt[4]{\left(w_{j}^{\rho}\right)^{2} \cdot\left(w_{i}^{\sigma}\right)^{2}}\right), i \neq j .\end{cases}$

\section{Proof}


1. First, we prove that $G_{p}=\left(\tilde{g}_{i j}\right)_{n \times n}$ is a PFPR.

As $\tilde{g}_{i j}^{\rho}=\sqrt[4]{\left(w_{i}^{\rho}\right)^{2} \cdot\left(w_{j}^{\sigma}\right)^{2}}=\sqrt[4]{\left(w_{j}^{\sigma}\right)^{2} \cdot\left(w_{i}^{\rho}\right)^{2}}=\tilde{g}_{j i}^{\sigma}$ can be obtained by Eq. (11) for all $i, j \in N$.

Then, since $w_{i}^{\rho}, w_{j}^{\sigma} \in[0,1]$ and $\left(w_{i}^{\rho}\right)^{2}+\left(w_{j}^{\sigma}\right)^{2} \leq 1$, we know the expression of $0 \leq \sqrt[4]{\left(w_{i}^{\rho}\right)^{2} \cdot\left(w_{j}^{\sigma}\right)^{2}} \leq 1,0 \leq \sqrt[4]{\left(w_{i}^{\sigma}\right)^{2} \cdot\left(w_{j}^{\rho}\right)^{2}} \leq 1, i \neq j$, which can be denoted as $0 \leq \tilde{g}_{i j}^{\rho} \leq 1,0 \leq \tilde{g}_{i j}^{\sigma} \leq 1$, and $i, j=1,2, \ldots, n$.

Simultaneously, it can be deduced that the following equation holds: $\left(w_{i}^{\rho}\right)^{2}+\left(w_{i}^{\sigma}\right)^{2} \leq 1$

a $\mathrm{n} \quad \mathrm{d}$

$\sum_{j \neq i}^{n}\left(w_{j}^{\rho}\right)^{2} \leq\left(w_{i}^{\sigma}\right)^{2}, \sum_{j \neq i}^{n}\left(w_{j}^{\sigma}\right)^{2} \leq\left(w_{i}^{\rho}\right)^{2}+n-2, \quad i=1,2, \ldots, n$.

\section{Models for constructing a multiplicative consistent PFPR}

In this complex world, when handling decision-making problems with certain complexity and uncertainty, it is unimaginably difficult for decision-makers to establish PFPRs with multiplicative consistency. Therefore, Eq. (3.10) cannot be established, namely, at least one of the following cases exists:

$$
\begin{aligned}
\left(\tilde{g}_{i j}^{\rho}\right)^{2}+\left(\tilde{g}_{i j}^{\sigma}\right)^{2} & =\sqrt{\left(w_{i}^{\rho}\right)^{2} \cdot\left(w_{j}^{\sigma}\right)^{2}}+\sqrt{\left(w_{i}^{\sigma}\right)^{2} \cdot\left(w_{j}^{\rho}\right)^{2}} \leq \frac{\left(w_{i}^{\rho}\right)^{2}+\left(w_{j}^{\sigma}\right)^{2}}{2}+\frac{\left(w_{i}^{\sigma}\right)^{2}+\left(w_{j}^{\rho}\right)^{2}}{2} \\
& =\frac{\left[\left(w_{i}^{\rho}\right)^{2}+\left(w_{i}^{\sigma}\right)^{2}\right]+\left[\left(w_{j}^{\sigma}\right)^{2}+\left(w_{j}^{\rho}\right)^{2}\right]}{2} \leq \frac{1+1}{2}=1 .
\end{aligned}
$$

Therefore, $G_{p}=\left(\tilde{g}_{i j}\right)_{n \times n}$ is a PFPR.

2. Second, we prove that PFPR $G_{p}$ is a multiplicatively consistent.

As for all $i, j, k=1,2, \ldots, n$, the following equation $\rho_{i j} \neq \sqrt[4]{\left(w_{i}^{\rho}\right)^{2} \cdot\left(w_{j}^{\sigma}\right)^{2}}$, or $\sigma_{i j} \neq \sqrt[4]{\left(w_{j}^{\rho}\right)^{2} \cdot\left(w_{i}^{\sigma}\right)^{2}}$. holds:

$$
\mu\left(\tilde{g}_{i k}\right) \cdot \mu\left(\tilde{g}_{k j}\right)=\frac{\left(\tilde{g}_{i k}^{\rho}\right)^{2}}{\left(\tilde{g}_{i k}^{\sigma}\right)^{2}} \cdot \frac{\left(\tilde{g}_{k j}^{\rho}\right)^{2}}{\left(\tilde{g}_{k j}^{\sigma}\right)^{2}}=\frac{\sqrt{w_{i}^{\rho} \cdot w_{k}^{\sigma}}}{\sqrt{w_{i}^{\sigma} \cdot w_{k}^{\rho}}} \cdot \frac{\sqrt{w_{k}^{\rho} \cdot w_{j}^{\sigma}}}{\sqrt{w_{k}^{\sigma} \cdot w_{j}^{\rho}}}=\frac{\sqrt{w_{i}^{\rho} \cdot w_{j}^{\sigma}}}{\sqrt{w_{i}^{\sigma} \cdot w_{j}^{\rho}}}=\frac{\left(\tilde{g}_{i j}^{\rho}\right)^{2}}{\left(\tilde{g}_{i j}^{\sigma}\right)^{2}}=\mu\left(\tilde{g}_{i j}\right) .
$$

Therefore, PFPR $G_{p}$ is a multiplicatively consistent PFPR in light of Theorem 1.

In summary, this completes the proof of Theorem 2.

Corollary 1 Assume that $P=\left(\tilde{p}_{i j}\right)_{n \times n}=\left(\left\langle\rho_{i j}, \sigma_{i j}\right\rangle\right)_{n \times n}$ is a PFPR. If there is a standardized Pythagorean fuzzy weight vector $w=\left\{w_{1}, w_{2}, \ldots, w_{n}\right\}^{T}$ with the following condition: $\tilde{p}_{i j}=\left(\rho_{i j}, \sigma_{i j}\right)= \begin{cases}(\sqrt{2} / 2, \sqrt{2} / 2), & i=j, \\ \left(\sqrt[4]{\left(w_{i}^{\rho}\right)^{2} \cdot\left(w_{j}^{\sigma}\right)^{2}}, \sqrt[4]{\left(w_{j}^{\rho}\right)^{2} \cdot\left(w_{i}^{\sigma}\right)^{2}}\right), & i \neq j .\end{cases}$

Then, $P=\left(\tilde{p}_{i j}\right)_{n \times n}=\left(\left\langle\rho_{i j}, \sigma_{i j}\right\rangle\right)_{n \times n}$ is a multiplicatively consistent PFPR, where $w_{i} \equiv\left(w_{i}^{\rho}, w_{i}^{\sigma}\right), 0 \leq w_{i}^{\rho}, w_{i}^{\sigma} \leq 1$,
Case 1: If $\rho_{i j} \neq \sqrt[4]{\left(w_{i}^{\rho}\right)^{2} \cdot\left(w_{j}^{\sigma}\right)^{2}}$, one can obtain $\ln \rho_{i j} \neq \ln \sqrt[4]{\left(w_{i}^{\rho}\right)^{2} \cdot\left(w_{j}^{\sigma}\right)^{2}}$. Hence, one can see that

$\ln \rho_{i j} \neq 0.25 \ln \left(w_{i}^{\rho}\right)^{2}+0.25 \ln \left(w_{j}^{\sigma}\right)^{2}$.

Next, we introduce two non-negative deviation variables $d_{i j}^{-}$and $d_{i j}^{+}$into the equation, which are expressed as follows $d_{i j}^{-}-d_{i j}^{+}=0.25 \ln \left(w_{i}^{\rho}\right)^{2}+0.25 \ln \left(w_{j}^{\sigma}\right)^{2}-\ln \rho_{i j}, \quad i, j=1,2, \ldots, n$,

where $d_{i j}^{-} \cdot d_{i j}^{+}=0 . \quad i, j=1,2, \ldots, n$. 
Obviously, the smaller the two non-negative deviation variables $d_{i j}^{-}, d_{i j}^{+}$are, the better the multiplicative consistency. Therefore, the optimization model can be constructed to generate the standardized Pythagorean fuzzy weight vector, which minimizes the sum of two non-negative deviation variables $e_{i j}^{-}-e_{i j}^{+}=0.25 \ln \left(w_{i}^{\sigma}\right)^{2}+0.25 \ln \left(w_{j}^{\rho}\right)^{2}-\ln \sigma_{i j}, i, j=1,2, \ldots, n$,

where $e_{i j}^{-} \cdot e_{i j}^{+}=0 . i, j=1,2, \ldots, n$.

Similarly, the smaller the two non-negative deviation variables $e_{i j}^{-}$and $e_{i j}^{+}$are, the better the multiplicative consist-

(M-3.1)

$$
s . t \begin{cases}d_{i j}^{-}-d_{i j}^{+}=0.25 \ln \left(\tilde{w}_{i}^{\rho}\right)^{2}+0.25 \ln \left(\tilde{w}_{j}^{\sigma}\right)^{2}-\ln \rho_{i j}, & i, j=1,2, \ldots, n, \\ d_{i j}^{-}>0, d_{i j}^{+}>0, d_{i j}^{-} \cdot d_{i j}^{+}=0, & i, j=1,2, \ldots, n \\ 0 \leq w_{i}^{\rho} \leq 1,0 \leq w_{i}^{\sigma} \leq 1,\left(w_{i}^{\rho}\right)^{2}+\left(w_{i}^{\sigma}\right)^{2} \leq 1, & i=1,2, \ldots, n, \\ \sum_{j \neq i}^{n}\left(w_{j}^{\rho}\right)^{2} \leq\left(w_{i}^{\sigma}\right)^{2}, \sum_{j \neq i}^{n}\left(w_{j}^{\sigma}\right)^{2} \leq\left(w_{i}^{\rho}\right)^{2}+n-2, & i=1,2, \ldots, n .\end{cases}
$$

Case 2: If $\sigma_{i j} \neq \sqrt[4]{\left(w_{j}^{\rho}\right)^{2} \cdot\left(w_{i}^{\sigma}\right)^{2}}$, we introduce two nonnegative deviation variables $e_{i j}^{-}$and $e_{i j}^{+}$into the equation, which is expressed as follows: ency. Therefore, we can establish an optimization model and minimize the sum of the two non-negative deviation variables to obtain the normalized Pythagorean fuzzy weight vector

(M-3.2) $\quad \min \delta_{2}=\sum_{i=1}^{n} \sum_{j=i}^{n}\left(e_{i j}^{-}+e_{i j}^{+}\right)$

$$
\text { s.t } \begin{cases}e_{i j}^{-}-e_{i j}^{+}=0.25 \ln \left(w_{i}^{\sigma}\right)^{2}+0.25 \ln \left(w_{j}^{\rho}\right)^{2}-\ln \sigma_{i j}, & i, j=1,2, \ldots, n, \\ e_{i j}^{-}>0, e_{i j}^{+}>0, e_{i j}^{-} \cdot e_{i j}^{+}=0, & i, j=1,2, \ldots, n, \\ 0 \leq w_{i}^{\rho} \leq 1,0 \leq w_{i}^{\sigma} \leq 1,\left(w_{i}^{\rho}\right)^{2}+\left(w_{i}^{\sigma}\right)^{2} \leq 1, & i=1,2, \ldots, n, \\ \sum_{j \neq i}^{n}\left(w_{j}^{\rho}\right)^{2} \leq\left(w_{i}^{\sigma}\right)^{2}, \sum_{j \neq i}^{n}\left(w_{j}^{\sigma}\right)^{2} \leq\left(w_{i}^{\rho}\right)^{2}+n-2, & i=1,2, \ldots, n .\end{cases}
$$

(M-3.3) $\min \bar{\delta}=\delta_{1}+\delta_{2}=\sum_{i=1}^{n} \sum_{j=i}^{n}\left(d_{i j}^{-}+d_{i j}^{+}+e_{i j}^{-}+e_{i j}^{+}\right)$

$$
s . t \begin{cases}d_{i j}^{-}-d_{i j}^{+}=0.25 \ln \left(w_{i}^{\rho}\right)^{2}+0.25 \ln \left(w_{j}^{\sigma}\right)^{2}-\ln \rho_{i j}, & i, j=1,2, \ldots, n, \\ e_{i j}^{-}-e_{i j}^{+}=0.25 \ln \left(w_{i}^{\sigma}\right)^{2}+0.25 \ln \left(w_{j}^{\rho}\right)^{2}-\ln \sigma_{i j}, & i, j=1,2, \ldots, n, \\ d_{i j}^{-}>0, d_{i j}^{+}>0, d_{i j}^{-} \cdot d_{i j}^{+}=0, & i, j=1,2, \ldots, n, \\ e_{i j}^{-}>0, e_{i j}^{+}>0, e_{i j}^{-} \cdot e_{i j}^{+}=0, & i, j=1,2, \ldots, n, \\ 0 \leq w_{i}^{\rho} \leq 1,0 \leq w_{i}^{\sigma} \leq 1,\left(w_{i}^{\rho}\right)^{2}+\left(w_{i}^{\sigma}\right)^{2} \leq 1, & i=1,2, \ldots, n, \\ \sum_{j \neq i}^{n}\left(w_{j}^{\rho}\right)^{2} \leq\left(w_{i}^{\sigma}\right)^{2}, \sum_{j \neq i}^{n}\left(w_{j}^{\sigma}\right)^{2} \leq\left(w_{i}^{\rho}\right)^{2}+n-2, & i=1,2, \ldots, n .\end{cases}
$$


From Definition 4, we know $\rho_{i j}=\sigma_{j i}, \sigma_{i j}=\rho_{j i}$. Then,

2. Support that $\tilde{\delta}=0$ and $i, j \in N$, we can derive

$d_{i j}^{-}-d_{i j}^{+}=0.25 \ln \left(\tilde{w}_{i}^{\rho}\right)^{2}+0.25 \ln \left(w_{j}^{\sigma}\right)^{2}-\ln \rho_{i j}=0.25 \ln \left(w_{j}^{\sigma}\right)^{2}+0.25 \ln \left(w_{i}^{\rho}\right)^{2}-\ln \sigma_{j i}=e_{j i}^{-}-e_{j i}^{+}$.

Consequently, M-4.3 can be transformed into the model of M-4.4 by considering the upper triangular elements of PFPRs, which are expressed as follows:

$$
\tilde{\delta}=\sum_{i=1}^{n-1} \sum_{j=i+1}^{n}\left(d_{i j}^{-}+d_{i j}^{+}+e_{i j}^{-}+e_{i j}^{+}\right)=0 .
$$

A $s$

$d_{i j}^{-}, d_{i j}^{+}, e_{i j}^{-}, e_{i j}^{+}>0, d_{i j}^{-}=d_{i j}^{+}=e_{i j}^{-}=e_{i j}^{+}=0$ can be

(M-3.4) $\min \delta=\sum_{i=1}^{n-1} \sum_{j=i+1}^{n}\left(d_{i j}^{-}+d_{i j}^{+}+e_{i j}^{-}+e_{i j}^{+}\right)$

$$
\text { s.t } \begin{cases}d_{i j}^{-}-d_{i j}^{+}=0.25 \ln \left(w_{i}^{\rho}\right)^{2}+0.25 \ln \left(w_{j}^{\sigma}\right)^{2}-\ln \rho_{i j}, & i=1,2, \cdots, n-1, j=i+1, \cdots, n, \\ e_{i j}^{-}-e_{i j}^{+}=0.25 \ln \left(w_{i}^{\sigma}\right)^{2}+0.25 \ln \left(w_{j}^{\rho}\right)^{2}-\ln \sigma_{i j}, & i=1,2, \cdots, n-1, j=i+1, \cdots, n, \\ d_{i j}^{-}>0, d_{i j}^{+}>0, d_{i j}^{-} \cdot d_{i j}^{+}=0, & i=1,2, \cdots, n-1, j=i+1, \cdots, n, \\ e_{i j}^{-}>0, e_{i j}^{+}>0, e_{i j}^{-} \cdot e_{i j}^{+}=0, & i=1,2, \cdots, n-1, j=i+1, \cdots, n, \\ 0 \leq w_{i}^{\rho} \leq 1,0 \leq w_{i}^{\sigma} \leq 1,\left(w_{i}^{\rho}\right)^{2}+\left(w_{i}^{\sigma}\right)^{2} \leq 1, & i=1,2, \cdots, n, \\ \sum_{j \neq i}^{n}\left(w_{j}^{\rho}\right)^{2} \leq\left(w_{i}^{\sigma}\right)^{2}, \sum_{j \neq i}^{n}\left(w_{j}^{\sigma}\right)^{2} \leq\left(w_{i}^{\rho}\right)^{2}+n-2, & i=1,2, \cdots, n .\end{cases}
$$

Theorem 3 A PFPR P $=\left(\tilde{p}_{i j}\right)_{n \times n}=\left(\left\langle\rho_{i j}, \sigma_{i j}\right\rangle\right)_{n \times n}$ is a multiplicatively consistent PFPR if and only if $\tilde{\delta}=0$, where $\tilde{\delta}$ denotes the optimal objective value of the M-4.4.

\section{Proof}

1. Assuming that PFPR $P=\left(\tilde{p}_{i j}\right)_{n \times n}=\left(\left\langle\rho_{i j}, \sigma_{i j}\right\rangle\right)_{n \times n}$ is multiplicatively consistent, one can obtain $\rho_{i j}=\sqrt[4]{\left(w_{i}^{\rho}\right)^{2} \cdot\left(w_{j}^{\sigma}\right)^{2}}$ and $\sigma_{i j}=\sqrt[4]{\left(w_{j}^{\rho}\right)^{2} \cdot\left(w_{i}^{\sigma}\right)^{2}}$, $i, j=1,2, \ldots, n$ from Eq. (14) in Corollary 1 . We can express that $\ln \rho_{i j}=0.25 \ln \left(w_{i}^{\rho}\right)^{2}+0.25 \ln \left(w_{j}^{\sigma}\right)^{2}$, $\ln \sigma_{i j} 0.25 \ln \left(w_{i}^{\sigma}\right)^{2}+0.25 \ln \left(w_{j}^{\rho}\right)^{2}$, and $i, j=1,2, \ldots, n$.

In addition, according to Eq. (4.9), one can obtain $d_{i j}^{-}=d_{i j}^{+}=e_{i j}^{-}=e_{i j}^{+}=0, i, j=1,2, \ldots, n$.

$\mathrm{Th}$ e refore, we $\tilde{\delta}=\sum_{i=1}^{n-1} \sum_{j=i+1}^{n}\left(d_{i j}^{-}+d_{i j}^{+}+e_{i j}^{-}+e_{i j}^{+}\right)=0$.

$\hat{\rho}_{i j}=\left\{\begin{array}{ll}\rho_{i j} \cdot e^{d_{i j}^{-}}, & (i, j) \in n \times n, d_{i j}^{-}>0, d_{i j}^{+}=0, \\ \rho_{i j}, \quad(i, j) \in n \times n, d_{i j}^{-}=0, d_{i j}^{+}=0, \\ \rho_{i j} \cdot e^{d_{i j}^{+}}, \quad(i, j) \in n \times n, d_{i j}^{-}=0, d_{i j}^{+}>0,\end{array} \quad \hat{\sigma}_{i j}= \begin{cases}\sigma_{i j} \cdot e^{d_{i j}^{-}}, & (i, j) \in n \times n, e_{i j}^{-}>0, e_{i j}^{+}=0, \\ \sigma_{i j}, \quad(i, j) \in n \times n, e_{i j}^{-}=0, e_{i j}^{+}=0, & \\ \sigma_{i j} \cdot e^{d_{i j}^{+}}, & (i, j) \in n \times n, e_{i j}^{-}=0, e_{i j}^{+}>0 .\end{cases}\right.$ deduced.

Through the above proof, we can see that there is a normalized Pythagorean fuzzy weight vector $w=\left\{w_{1}, w_{2}, \ldots, w_{n}\right\}^{T}$, and the elements in the PFPR can be expressed by Eq. (14). According to Corollary 1 , the PFPR $P=\left(\tilde{p}_{i j}\right)_{n \times n}=\left(\left\langle\rho_{i j}, \sigma_{i j}\right\rangle\right)_{n \times n}$ is multiplicatively consistent.

Therefore, the proof is completed.

One can obtain that the optimal Pythagorean fuzzy weight vectors $\tilde{w}=\left(\tilde{w}_{1}, \tilde{w}_{2}, \ldots, \tilde{w}_{n}\right)^{T}=\left(\left\langle\tilde{w}_{1}^{\rho}, \tilde{w}_{1}^{\sigma}\right\rangle,\left\langle\tilde{w}_{2}^{\rho}, \tilde{w}_{2}^{\sigma}\right\rangle, \ldots\left\langle\tilde{w}_{n}^{\rho}, \tilde{w}_{n}^{\sigma}\right\rangle\right)^{T}$ and the optimal deviation variables $\tilde{d}_{i j}^{-}, \tilde{d}_{i j}^{+}, \tilde{e}_{i j}^{-}, \tilde{e}_{i j}^{+}$.

When the optimal objective value $\tilde{\delta}=0$, $d_{i j}^{-}=d_{i j}^{+}=e_{i j}^{-}=e_{i j}^{+}=0$ is considered equally, where $i, j \in N$. That is, PFPR $P=\left(\tilde{p}_{i j}\right)_{n \times n}=\left(\left\langle\rho_{i j}, \sigma_{i j}\right\rangle\right)_{n \times n}$ is multiplicatively consistent. Otherwise, we can improve the consistency of PFPRs using the following methods: 
Theorem 4 PFPR $\hat{P}=\left(\hat{p}_{i j}\right)_{n \times n}=\left(\left\langle\hat{\rho}_{i j}, \hat{\sigma}_{i j}\right\rangle\right)_{n \times n}$ is a multiplicatively consistent PFPR, in which the elements satisfy Eq. (4.10).

Proof Based on the content mentioned above, we can know

\section{Multiplicative consistency improving algorithm}

In general, it is impractical to derive the conditions for complete multiplicatively consistent PFPRs. Therefore, the

$$
\left\{\begin{array} { l } 
{ d _ { i j } ^ { - } - d _ { i j } ^ { + } = 0 . 2 5 \operatorname { l n } ( w _ { i } ^ { \rho } ) ^ { 2 } + 0 . 2 5 \operatorname { l n } ( w _ { j } ^ { \sigma } ) ^ { 2 } - \operatorname { l n } \rho _ { i j } , } \\
{ e _ { i j } ^ { - } - e _ { i j } ^ { + } = 0 . 2 5 \operatorname { l n } ( w _ { i } ^ { \sigma } ) ^ { 2 } + 0 . 2 5 \operatorname { l n } ( w _ { j } ^ { \rho } ) ^ { 2 } - \operatorname { l n } \sigma _ { i j } , }
\end{array} \Rightarrow \left\{\begin{array}{l}
\ln \rho_{i j}+d_{i j}^{-}-d_{i j}^{+}=0.25 \ln \left(w_{i}^{\rho}\right)^{2}+0.25 \ln \left(w_{j}^{\sigma}\right)^{2}, \\
\ln \sigma_{i j}+e_{i j}^{-}-e_{i j}^{+}=0.25 \ln \left(w_{i}^{\sigma}\right)^{2}+0.25 \ln \left(w_{j}^{\rho}\right)^{2} .
\end{array}\right.\right.
$$

$\left\{\begin{array}{l}\mathrm{T} \rho_{i j} \cdot e^{d_{i j}^{-}-d_{i j}^{+}}=\sqrt[4]{\left(w_{i}^{\rho}\right)^{2} \cdot\left(w_{j}^{\sigma}\right)^{2}} \\ \sigma_{i j} \cdot e^{e_{i j}^{-}-e_{i j}^{+}}=\sqrt[4]{\left(w_{i}^{\sigma}\right)^{2} \cdot\left(w_{j}^{\rho}\right)^{2}}\end{array} \Rightarrow\left\{\begin{array}{l}\hat{\rho}_{i j}=\sqrt[4]{\left(\hat{w}_{i}^{\rho}\right)^{2} \cdot\left(\hat{w}_{j}^{\sigma}\right)^{2}} \\ \hat{\sigma}_{i j}=\sqrt[4]{\left(\hat{w}_{i}^{\sigma}\right)^{2} \cdot\left(\hat{w}_{j}^{\rho}\right)^{2}}\end{array}\right.\right.$,

where $i, j=1,2, \ldots, n$.

Furthermore, an algorithm that obtains the multiplicatively consistent PFPR by aggregating the optimal weight values is proposed, as shown below.

Step 1: Construct the PFPR matrix $P=\left(\tilde{p}_{i j}\right)_{n \times n}=\left(\left\langle\rho_{i j}, \sigma_{i j}\right\rangle\right)_{n \times n}$ from decision-making information given by the DM.

Step 2: Determine whether the PFPR matrix $P$ is ordered consistent with Definition 7. If yes, the ranking result will be obtained directly. Otherwise, please proceed to the next step 3.

Step 3: The optimal standardized Pythagorean fuzzy weight vectors and deviation variables are obtained by solving the optimal model in connection with the decision-making problem.

Step 4: Calculate the score functions $s\left(\tilde{w}_{i}\right)$ and accuracy functions $h\left(\tilde{w}_{i}\right)$ by the following equations: $s\left(\tilde{w}_{i}\right)=\left(\tilde{w}_{i}^{\rho}\right)^{2}-\left(\tilde{w}_{i}^{\sigma}\right)^{2}$ and $h\left(\tilde{w}_{i}\right)=\left(\tilde{w}_{i}^{\rho}\right)^{2}+\left(\tilde{w}_{i}^{\sigma}\right)^{2}$, where $i=1,2, \ldots, n$.

Step 5: Rank the standardized Pythagorean fuzzy weight vectors $\tilde{w}_{i}$ according to the comparison method mentioned above in Definition 8.

Step 6: Determine the optimum alternative in terms of ranking results.

Step 7: Form a PFPR $\hat{P}=\left(\hat{p}_{i j}\right)_{n \times n}=\left(\left\langle\hat{\rho}_{i j}, \hat{\sigma}_{i j}\right\rangle\right)_{n \times n}$ with complete multiplicative consistency based on the improved equations. consistency index $(\mathrm{CI})$ will be introduced to measure the individual consistency of PFPRs.

Definition 10 Suppose that $A=\left(a_{i j}\right)_{n \times n}=\left(\left\langle a_{i j, \rho}, a_{i j, \sigma}\right\rangle\right)_{n \times n}$ is a PFPR and that $\tilde{A}=\left(\tilde{a}_{i j}\right)_{n \times n}=\left(\left\langle\tilde{a}_{i j, \rho}, \tilde{a}_{i j, \sigma}\right\rangle\right)_{n \times n}$ is a multiplicatively consistent PFPR. The consistency index (CI) is denoted by

$$
\begin{aligned}
C I(A)= & \frac{1}{n(n-1)} \sum_{\substack{i, j=1 \\
i<j}}^{n}\left(\left|\ln \left(a_{i j, \rho}\right)-\ln \left(\hat{a}_{i j, \rho}\right)\right|\right. \\
& \left.+\left|\ln \left(a_{i j, \sigma}\right)-\ln \left(\hat{a}_{i j, \sigma}\right)\right|\right) .
\end{aligned}
$$

Obviously, the smaller $C I(A)$ is, the higher the degree of consistency of $A$. $A$ is fully multiplicatively consistent when the condition $C I(A)=0$ is satisfied.

Definition 11 Suppose that PFPR $A=\left(a_{i j}\right)_{n \times n}=\left(\left\langle a_{i j, \rho}, a_{i j, \sigma}\right\rangle\right)_{n \times n}$ and $\overline{C I}$ is the given consistency index threshold. $A$ is called acceptably multiplicatively consistent PFPR, which is expressed as $C I(A) \leq \overline{C I}$.

To better improve the consistency of the PFPRs given by experts, the consistency improving algorithm (A-3.2) is constructed below. Through the effective improvement of the algorithm (A-3.2), the adjusted PFPRs can reach the preset consistency index threshold, increasing the reliability of the decision result.

\section{(A-3.2)}

Input: Individual PFPRs $A=\left(a_{i j}\right)_{n \times n}=\left(\left\langle a_{i j, \rho}, a_{i j, \sigma}\right\rangle\right)_{n \times n}$, consistency threshold $\overline{C I}$, and parameter $\gamma$.

Output: Acceptably multiplicatively consistent PFPR $A^{*}$, consistency index $C I\left(A^{*}\right)$, iterations $l$, and optimum alternative $x^{*}$. 
Step 1: Let $A^{l}=\left(a_{i j}^{l}\right)_{n \times n}=A=\left(a_{i j}\right)_{n \times n}$, where $l=0$.

Step 2: The optimal standardized Pythagorean fuzzy weight vectors $\hat{w}^{l}=\left\{\hat{w}_{1}^{l}, \hat{w}_{2}^{l}, \ldots, \hat{w}_{n}^{l}\right\}^{T}$ and deviation variables $\hat{d}_{i j}^{l-}, \hat{d}_{i j}^{l+}, \hat{e}_{i j}^{l-}, \hat{e}_{i j}^{l+}$ are obtained by solving the optimal model in connection with the decision-making problem.

Step 3: Construct a multiplicatively consistent PFPR matrix $\hat{A}^{l}=\left(\hat{a}_{i j}^{l}\right)_{n \times n}=\left(\left\langle\hat{a}_{i j, \rho}^{l}, \hat{a}_{i j, \sigma}^{l}\right\rangle\right)_{n \times n}$, where

$\hat{a}_{i j, \rho}^{l}=\left\{\begin{array}{ll}a_{i j, \rho}^{l} \cdot e^{\tilde{d}_{i j}^{l-}-\tilde{d}_{i j}^{l+}}, & i \neq j, \\ \sqrt{2} / 2, & i=j,\end{array} \quad \hat{a}_{i j, \sigma}^{l}= \begin{cases}a_{i j, \sigma}^{l} \cdot e^{\tilde{d}_{i j}^{l-}-\tilde{d}_{i j}^{l+}}, & i \neq j, \\ \sqrt{2} / 2, & i=j .\end{cases}\right.$

Step 4: Calculate $\quad C I\left(A^{l}\right), \quad$ where $C I(A)=\frac{1}{n(n-1)} \sum_{\substack{i, j=1 \\ i<j}}^{n}\left(\left|\ln \left(a_{i j, \rho}\right)-\ln \left(\hat{a}_{i j, \rho}\right)\right|+\left|\ln \left(a_{i j, \sigma}\right)-\ln \left(\hat{a}_{i j, \sigma}\right)\right|\right)$. If $C I(A) \leq \overline{C I}$, which indicates that $A^{l}=\left(a_{i j}^{l}\right)_{n \times n}$ is the acceptable multiplicative consistency, continue to step 6 . Otherwise, go to step 5 .

Step 5: Let the iterative PFPR $A^{l+1}=\left(a_{i j}^{l+1}\right)_{n \times n}=\left(\left\langle a_{i j, \rho}^{l+1}, a_{i j, \sigma}^{l+1}\right\rangle\right)_{n \times n}$ and $l=l+1$ for all $i, j \in N, \quad$ where $\quad a_{i j, \rho}^{l+1}=\left(a_{i j, \rho}^{l}\right)^{1-\gamma} \cdot\left(\hat{a}_{i j, \rho}^{l}\right)^{\gamma} \quad$ and $\hat{a}_{i j, \sigma}^{l+1}=\left(a_{i j, \sigma}^{l}\right)^{1-\gamma} \cdot\left(\hat{a}_{i j, \sigma}^{l}\right)^{\gamma}$. Then, return to step 2.
Step 6: Let the acceptable multiplicatively consistency $\bar{A}=A^{l}$, whose consistency index is $C I(\bar{A})$ and $w^{*}=\hat{w}^{l}$.

Step 7: The weights are sorted by calculating the score function $s^{\prime}\left(w_{i}^{*}\right)$ and accuracy function $h^{\prime}\left(w_{i}^{*}\right)$, and then, the best alternative $x^{*}$ is selected.

Step 8: End.

Theorem 5 (Convergence) Assuming that PFPR $A=\left(a_{i j}\right)_{n \times n}$ and $\operatorname{CI}\left(A^{(l)}\right)$ is a consistency index of $A^{(l)}$, one can obtain

$C I\left(A^{(l+1)}\right)<C I\left(A^{(l)}\right), \lim _{t \rightarrow+\infty} C I\left(A^{(l)}\right)=0$,

where $l$ is the number of iterations.

Proof From the content mentioned above, we can know

$\begin{cases}d_{i j}^{(l)-}-d_{i j}^{(l)+}=0.25 \ln \left(w_{i, \rho}^{(l)}\right)^{2}+0.25 \ln \left(w_{j, \sigma}^{(l)}\right)^{2}-\ln \rho_{i j}^{(l)}, & i<j, \\ e_{i j}^{(l)-}-e_{i j}^{(l)+}=0.25 \ln \left(w_{i, \sigma}^{(l)}\right)^{2}+0.25 \ln \left(w_{j, \rho}^{(l)}\right)^{2}-\ln \sigma_{i j}^{(l)}, & i<j .\end{cases}$

S i n c e $\quad d_{i j}^{(l)-}, d_{i j}^{(l)+}, e_{i j}^{(l)-}, e_{i j}^{(l)+} \geq 0 \quad$ a n d $d_{i j}^{(l)-} \cdot d_{i j}^{(l)+}=0, e_{i j}^{(l)-} \cdot e_{i j}^{(l)+}=0, \quad$ two expressions of $d_{i j}^{(l)-}+d_{i j}^{(l)+}=\left|d_{i j}^{(l)+}-d_{i j}^{(l)-}\right|$ and $e_{i j}^{(l)-}+e_{i j}^{(l)+}=\left|e_{i j}^{(l)+}-e_{i j}^{(l)-}\right|$ can be obtained.

Let PFPR $\hat{A}^{(l)}$ be multiplicatively consistent. With each iteration, we know

$\left\{\begin{array}{l}\hat{a}_{i j, \rho}^{l}=\sqrt[4]{\left(\hat{w}_{i, \rho}^{(l)}\right)^{2} \cdot\left(\hat{w}_{j, \sigma}^{(l)}\right)^{2}}, i \neq j, \\ \hat{a}_{i j, \sigma}^{l}=\sqrt[4]{\left(\hat{w}_{i, \sigma}^{(l)}\right)^{2} \cdot\left(\hat{w}_{j, \rho}^{(l)}\right)^{2}}, i \neq j,\end{array} \Rightarrow\left\{\begin{array}{l}\ln \hat{a}_{i j, \rho}^{l}=0.25 \ln \left(\hat{w}_{i, \rho}^{(l)}\right)^{2}+0.25 \ln \left(\hat{w}_{j, \sigma}^{(l)}\right)^{2}, i \neq j, \\ \ln \hat{a}_{i j, \sigma}^{l}=0.25 \ln \left(\hat{w}_{i, \sigma}^{(l)}\right)^{2}+0.25 \ln \left(\hat{w}_{j, \rho}^{(l)}\right)^{2}, i \neq j .\end{array}\right.\right.$

Here, $\hat{d}_{i j}^{l-}, \hat{d}_{i j}^{l+}, \hat{e}_{i j}^{l-}, \hat{e}_{i j}^{l+}$ are the deviation variables and the optimal standardized Pythagorean fuzzy weight vectors $\hat{w}^{l}=\left\{\hat{w}_{1}^{l}, \hat{w}_{2}^{l}, \ldots, \hat{w}_{n}^{l}\right\}^{T}$, where $i, j=1,2, \ldots, n$ 


$$
\begin{aligned}
C I\left(A^{(l+1)}\right) & =\frac{1}{n(n-1)} \sum_{\substack{i, j=1 \\
i<j}}^{n}\left(\left|\ln \left(a_{i j, \rho}^{(l+1)}\right)-\ln \left(\hat{a}_{i j, \rho}^{(l+1)}\right)\right|+\left|\ln \left(a_{i j, \sigma}^{(l+1)}\right)-\ln \left(\hat{a}_{i j, \sigma}^{(l+1)}\right)\right|\right) \\
& =\frac{1}{n(n-1)} \sum_{\substack{i, j=1 \\
i<j}}^{n}\left(\left|\hat{d}_{i j}^{(l+1)-}-\hat{d}_{i j}^{(l+1)+}\right|+\left|\hat{e}_{i j}^{(l+1)-}-\hat{e}_{i j}^{(l+1)+}\right|\right) \\
& =\frac{1}{n(n-1)} \sum_{\substack{i, j=1 \\
i<j}}^{n}\left(\hat{d}_{i j}^{(l+1)-}+\hat{d}_{i j}^{(l+1)+}+\hat{e}_{i j}^{(l+1)-}+\hat{e}_{i j}^{(l+1)+}\right)\left(\operatorname{let} w_{i}^{(l+1)}=\hat{w}_{i}^{(l)}\right) \\
& \leq \frac{1}{n(n-1)} \sum_{\substack{i, j=1 \\
i<j}}^{n}\left(\left|0.25 \ln \left(\hat{w}_{i, \rho}^{(l)}\right)^{2}+0.25 \ln \left(\hat{w}_{j, \sigma}^{(l)}\right)^{2}-\ln a_{i j, \rho}^{(l+1)}\right|+\left|0.25 \ln \left(\hat{w}_{i, \sigma}^{(l)}\right)^{2}+0.25 \ln \left(\hat{w}_{j, \rho}^{(l)}\right)^{2}-\ln a_{i j, \sigma}^{(l+1)}\right|\right) \\
& =\frac{1}{n(n-1)} \sum_{\substack{i, j=1 \\
i<j}}^{n}\left(\left|\ln \left(a_{i j, \rho}^{(l+1)}\right)-\ln \left(\hat{a}_{i j, \rho}^{(l)}\right)\right|+\left|\ln \left(a_{i j, \sigma}^{(l+1)}\right)-\ln \left(\hat{a}_{i j, \sigma}^{(l)}\right)\right|\right) \\
& =\frac{1}{n(n-1)} \sum_{\substack{i, j=1 \\
i<j}}^{n}\left(\left|(1-\gamma) \ln \left(a_{i j, \rho}^{(l)}\right)+\gamma \ln \left(\hat{a}_{i j, \rho}^{(l)}\right)-\ln \left(\hat{a}_{i j, \rho}^{(l)}\right)\right|+\left|(1-\gamma) \ln \left(a_{i j, \sigma}^{(l)}\right)+\gamma \ln \left(\hat{a}_{i j, \sigma}^{(l)}\right)-\ln \left(\hat{a}_{i j, \sigma}^{(l)}\right)\right|\right) \\
& =(1-\gamma) \frac{1}{n(n-1)} \sum_{i, j=1}^{n}\left(\left|\ln \left(a_{i j, \rho}^{(l)}\right)-\ln \left(\hat{a}_{i j, \rho}^{(l)}\right)\right|+\left|\ln \left(a_{i j, \sigma}^{(l)}\right)-\ln \left(\hat{a}_{i j, \sigma}^{(l)}\right)\right|\right) \\
& =(1-\gamma) C I\left(A^{(l)}\right)<C I\left(A^{(l)}\right) .
\end{aligned}
$$

where parameter $\gamma(0<\gamma<1)$ is used to adjust the iterations.

Moreover, another representation is also available as follows:

\section{Degree of consensus}

Definition 12 Let $A^{k}=\left(a_{i j}^{k}\right)_{n \times n}=\left(\left\langle a_{i j, \rho}^{k}, a_{i j, \sigma}^{k}\right\rangle\right)_{n \times n}(k \in m)$ be a set of PFPRs on the set $X=\left\{x_{1}, x_{2}, \ldots, x_{n}\right\} \cdot \operatorname{ICD}\left(A^{k}\right)$ is

$\lim _{l \rightarrow+\infty} C I\left(A^{(l)}\right) \leq \lim _{l \rightarrow+\infty}(1-\gamma) C I\left(A^{(l-1)}\right) \leq \lim _{l \rightarrow+\infty}(1-\gamma)^{2} C I\left(A^{(l-2)}\right) \leq \cdots \leq \lim _{l \rightarrow+\infty}(1-\gamma)^{l} C I\left(A^{(0)}\right)=0$.

Since $C I\left(A^{(l)}\right) \geq 0$, we have $\lim _{l \rightarrow+\infty} C I\left(A^{(l)}\right)=0$.

In conclusion, the proof of Theorem 5 is completed.

\section{Consensus reaching process with PFPRs}

The model construction in the previous section only focuses on an individual PFPR. However, in actual decision-making, DMs usually give multiple Pythagorean fuzzy preference relations. To address this, we will conduct further research. called the degree of individual consensus of PFPRs, which is expressed as

$$
\begin{aligned}
\operatorname{ICD}\left(A^{k}\right)= & \frac{1}{n(n-1)(m-1)} \sum_{i \neq k} \\
& \sum_{i<j}\left(\left|\ln a_{i j, \rho}^{k}-\ln a_{i j, \rho}^{t}\right|+\left|\ln a_{i j, \sigma}^{k}-\ln a_{i j, \sigma}^{t}\right|\right) .
\end{aligned}
$$

It should be noted that the smaller the value of $\operatorname{ICD}\left(A^{k}\right)$ is, the higher the consensus of $A^{k}$ with other PFPRs, which indicates the higher the consensus between a decision-maker and other decision-makers.

Definition 13 Let $A^{k}=\left(a_{i j}^{k}\right)_{n \times n}=\left(\left\langle a_{i j, \rho}^{k}, a_{i j, \sigma}^{k}\right\rangle\right)_{n \times n}(k \in m)$ be a set of PFPR on the set $X=\left\{x_{1}, x_{2}, \ldots, x_{n}\right\}$. $\operatorname{GCD}\left(A^{k}\right)$ is 
called the degree of group consensus of PFPRs, which is expressed as

$$
\begin{aligned}
& \mathrm{GCD}=\frac{1}{m} \sum_{k=1}^{m} \operatorname{ICD}\left(A^{k}\right) \\
&= \frac{1}{m n(n-1)(m-1)} \sum_{k=1}^{m} \sum_{i \neq k} \\
& \sum_{i<j}\left(\left|\ln a_{i j, \rho}^{k}-\ln a_{i j, \rho}^{t}\right|+\left|\ln a_{i j, \sigma}^{k}-\ln a_{i j, \sigma}^{t}\right|\right) .
\end{aligned}
$$

Similarly, the smaller $\operatorname{GCD}\left(A^{k}\right)$ is, the higher the group consensus of all PFPRs $A^{1}, A^{2}, \ldots, A^{m}$. That is, the higher the consensus of all decision-makers.

\section{Comprehensive PFPR}

Definition 14 Let $A^{k}=\left(a_{i j}^{k}\right)_{n \times n}=\left(\left\langle a_{i j, \rho}^{k}, a_{i j, \sigma}^{k}\right\rangle\right)_{n \times n}(k \in m)$ be a set of PFPR given by DMs $e^{k}(k \in m)$ on the set $X=\left\{x_{1}, x_{2}, \ldots, x_{n}\right\} . A^{c}$ is denoted as a comprehensive Pythagorean fuzzy preference relation, which is expressed as

$A^{c}=\left(a_{i j, \rho}^{c}, a_{i j, \sigma}^{c}\right)_{n \times n}=\left(\sqrt{\prod_{k=1}^{m}\left(\left(a_{i j, \rho}^{k}\right)^{2}\right)^{\lambda^{k}}}, \sqrt{\prod_{k=1}^{m}\left(\left(a_{i j, \sigma}^{k}\right)^{2}\right)^{\lambda^{k}}}, \quad i, j \in n\right.$,

where $\lambda=\left(\lambda_{1}, \lambda_{2}, \ldots, \lambda_{m}\right)^{T}$ is the corresponding weight vec- $\quad$ be obtained. From the one side of Eq. (30), we obtain tor of $e^{k}$ satisfying $\lambda^{k} \geq 0, k \in M$, and $\sum_{k=1}^{m} \lambda^{k}=1$.

$$
\left\{\begin{array}{l}
0 \leq a_{i j, \rho}^{c}=\sqrt{\prod_{k=1}^{m}\left(a_{i j, \rho}^{k}\right)^{\lambda^{k}}} \leq \sqrt{\sum_{k=1}^{m} \lambda^{k} a_{i j, \rho}^{k}} \leq \sqrt{\sum_{k=1}^{m} \lambda^{k}}=1, \\
0 \leq a_{i j, \sigma}^{c}=\sqrt{\prod_{k=1}^{m}\left(a_{i j, \sigma}^{k}\right)^{\lambda^{k}}} \leq \sqrt{\sum_{k=1}^{m} \lambda^{k} a_{i j, \sigma}^{k}} \leq \sqrt{\sum_{k=1}^{m} \lambda^{k}}=1,
\end{array} \Rightarrow 0 \leq a_{i j, \rho}^{c} \leq 1, \quad 0 \leq a_{i j, \sigma}^{c} \leq 1 .\right.
$$

Regarding the other side, $\left(a_{i j, \rho}^{c}\right)^{2}+\left(a_{i j, \sigma}^{c}\right)^{2} \leq 1$ is available in the following process:

$$
\begin{aligned}
\left(a_{i j, \rho}^{c}\right)^{2}+\left(a_{i j, \sigma}^{c}\right)^{2} & =\left(\sqrt{\prod_{k=1}^{m}\left(\left(a_{i j, \rho}^{k}\right)^{2}\right)^{\lambda^{k}}}\right)^{2}+\left(\sqrt{\prod_{k=1}^{m}\left(\left(a_{i j, \sigma}^{k}\right)^{2}\right)^{\lambda^{k}}}\right)^{2}=\prod_{k=1}^{m}\left(\left(a_{i j, \rho}^{k}\right)^{2}\right)^{\lambda^{k}}+\prod_{k=1}^{m}\left(\left(a_{i j, \sigma}^{k}\right)^{2}\right)^{\lambda^{k}} \\
& \leq \sum_{k=1}^{m} \lambda^{k}\left(\left(a_{i j, \sigma}^{k}\right)^{2}\right)^{\lambda^{k}}+\sum_{k=1}^{m} \lambda^{k}\left(a_{i j, \sigma}^{k}\right)^{2}=\sum_{k=1}^{m} \lambda^{k}\left(\left(a_{i j, \sigma}^{k}\right)^{2}+\left(a_{i j, \sigma}^{k}\right)^{2}\right)=1 .
\end{aligned}
$$

Therefore, we can deduce

$$
\left(a_{i j, \rho}^{c}\right)^{2}+\left(a_{i j, \sigma}^{c}\right)^{2} \leq 1, \quad i, j \in N
$$


To summarize, $A^{c}$ is a PFPR, which completes the proof.

However, in actual group decision-making, the conclusion of Theorem 6 has difficultly holding because of the different preferences of decision-makers. Therefore, we give further discussions on it.

Suppose that Corollary 1 is not valid, namely

$a_{i j, \rho}^{k} \neq \sqrt[4]{\left(w_{i}^{\rho}\right)^{2} \cdot\left(w_{j}^{\sigma}\right)^{2}}$

or

$a_{i j, \sigma}^{k} \neq \sqrt[4]{\left(w_{j}^{\rho}\right)^{2} \cdot\left(w_{i}^{\sigma}\right)^{2}}, \quad i \neq j, k \in m$.

$$
\begin{aligned}
d_{i j}^{k-}-d_{i j}^{k+}= & 0.25 \ln \left(w_{i}^{\rho}\right)^{2}+0.25 \ln \left(w_{j}^{\sigma}\right)^{2} \\
& -\ln a_{i j, \rho}^{k}, \quad i, j=1,2, \ldots, n, \\
e_{i j}^{k-}-e_{i j}^{k+}= & 0.25 \ln \left(w_{i}^{\sigma}\right)^{2}+0.25 \ln \left(w_{j}^{\rho}\right)^{2} \\
& -\ln a_{i j, \sigma}^{k}, \quad i, j=1,2, \ldots, n .
\end{aligned}
$$

Obviously, the smaller the non-negative deviation variables $d_{i j}^{k-}, d_{i j}^{k+}, e_{i j}^{k-}, e_{i j}^{k+}, i, j \in N, k \in M$ are, the better the multiplicative consistency. Therefore, the optimization model can be constructed to generate the standardized Pythagorean fuzzy weight vector, which minimizes the sum of two non-negative deviation variables

(M-4.1) $\min \Delta_{1}=\sum_{k=1}^{m} \sum_{i<j} \lambda^{k}\left(d_{i j}^{k-}+d_{i j}^{k+}+e_{i j}^{k-}+e_{i j}^{k+}\right)$

$$
\text { s.t } \begin{cases}d_{i j}^{k-}-d_{i j}^{k+}=0.25 \ln \left(w_{i}^{\rho}\right)^{2}+0.25 \ln \left(w_{j}^{\sigma}\right)^{2}-\ln a_{i j, \rho}^{k}, & i<j, k \in M, \\ e_{i j}^{k-}-e_{i j}^{k+}=0.25 \ln \left(w_{i}^{\sigma}\right)^{2}+0.25 \ln \left(w_{j}^{\rho}\right)^{2}-\ln a_{i j, \sigma}^{k}, & i<j, k \in M, \\ d_{i j}^{k-}>0, d_{i j}^{k+}>0, d_{i j}^{k-} \cdot d_{i j}^{k+}=0, & i<j, k \in M, \\ e_{i j}^{k-}>0, e_{i j}^{k+}>0, e_{i j}^{k-} \cdot e_{i j}^{k+}=0, & i<j, k \in M, \\ 0 \leq w_{i}^{\rho} \leq 1,0 \leq w_{i}^{\sigma} \leq 1,\left(w_{i}^{\rho}\right)^{2}+\left(w_{i}^{\sigma}\right)^{2} \leq 1, & i=1,2, \ldots, n, \\ \sum_{j \neq i}^{n}\left(w_{j}^{\rho}\right)^{2} \leq\left(w_{i}^{\sigma}\right)^{2}, \sum_{j \neq i}^{n}\left(w_{j}^{\sigma}\right)^{2} \leq\left(w_{i}^{\rho}\right)^{2}+n-2, & i=1,2, \ldots, n .\end{cases}
$$

Next, we introduce some non-negative deviation variables $d_{i j}^{k-}, d_{i j}^{k+}, e_{i j}^{k-}, e_{i j}^{k+}$ into the equation, which are expressed as follows:

$$
\begin{aligned}
& \mathrm{A} \\
& d_{i j}^{k-}-d_{i j}^{k+}=0.25 \ln \left(w_{i}^{\rho}\right)^{2}+0.25 \ln \left(w_{j}^{\sigma}\right)^{2}-\ln a_{i j, \rho}^{k}, e_{i j}^{k-} \\
& -e_{i j}^{k+}=0.25 \ln \left(w_{i}^{\sigma}\right)^{2}+0.25 \ln \left(w_{j}^{\rho}\right)^{2}-\ln a_{i j, \sigma}^{k} \quad \text { and } \\
& \sum_{k=1}^{m} \lambda^{k}=1 \text {, then }
\end{aligned}
$$

$\left\{\begin{array}{l}\sum_{k=1}^{m} \lambda^{k}\left(d_{i j}^{k-}-d_{i j}^{k+}\right)=0.25 \ln \left(w_{i}^{\rho}\right)^{2}+0.25 \ln \left(w_{j}^{\sigma}\right)^{2}-\sum_{k=1}^{m} \lambda^{k} \ln a_{i j, \rho}^{k}, i<j, \\ \sum_{k=1}^{m} \lambda^{k}\left(e_{i j}^{k-}-e_{i j}^{k+}\right)=0.25 \ln \left(w_{i}^{\sigma}\right)^{2}+0.25 \ln \left(w_{j}^{\rho}\right)^{2}-\sum_{k=1}^{m} \lambda^{k} \ln a_{i j, \sigma}^{k}, i<j .\end{array}\right.$

Combined with Eq. 4.3, one can obtain $\ln a_{i j, \rho}^{c}=\sum_{k=1}^{m} \lambda^{k} \ln a_{i j, \rho}^{k}, \ln a_{i j, \sigma}^{c}=\sum_{k=1}^{m} \lambda^{k} \ln a_{i j, \sigma}^{k}, i<j$. Let $d_{i j}^{c-}=\sum_{k=1}^{m} \lambda^{k} d_{i j}^{k-}, d_{i j}^{c++}=\sum_{k=1}^{m} \lambda^{k} d_{i j}^{k+}, e_{i j}^{c-}=\sum_{k=1}^{m} \lambda^{k} e_{i j}^{k-}$ and $e_{i j}^{c+}=\sum_{k=1}^{m} \lambda^{k} e_{i j}^{k+}$, and model (M-4.2) will be developed as the following optimization model: 
(M-4.2) $\quad \min \Delta_{2}=\sum_{i<j}\left(d_{i j}^{c-}+d_{i j}^{c+}+e_{i j}^{c-}+e_{i j}^{c+}\right)$

$$
\text { s.t } \begin{cases}d_{i j}^{c-}-d_{i j}^{c+}=0.25 \ln \left(w_{i}^{\rho}\right)^{2}+0.25 \ln \left(w_{j}^{\sigma}\right)^{2}-\ln a_{i j, \rho}^{c}, & i<j, \\ e_{i j}^{c-}-e_{i j}^{c+}=0.25 \ln \left(w_{i}^{\sigma}\right)^{2}+0.25 \ln \left(w_{j}^{\rho}\right)^{2}-\ln a_{i j, \sigma}^{c}, & i<j, \\ d_{i j}^{c-}>0, d_{i j}^{c+}>0, d_{i j}^{c-} \cdot d_{i j}^{c+}=0, & i<j, \\ e_{i j}^{c-}>0, e_{i j}^{c+}>0, e_{i j}^{c-} \cdot e_{i j}^{c+}=0, & i<j \\ 0 \leq w_{i}^{\rho} \leq 1,0 \leq w_{i}^{\sigma} \leq 1,\left(w_{i}^{\rho}\right)^{2}+\left(w_{i}^{\sigma}\right)^{2} \leq 1, & i=1,2, \ldots, n, \\ \sum_{j \neq i}^{n}\left(w_{j}^{\rho}\right)^{2} \leq\left(w_{i}^{\sigma}\right)^{2}, \sum_{j \neq i}^{n}\left(w_{j}^{\sigma}\right)^{2} \leq\left(w_{i}^{\rho}\right)^{2}+n-2, & i=1,2, \ldots, n .\end{cases}
$$

Therefore, one can be obtained that the optimal Pythagorean fuzzy weight vectors $\tilde{w}=\left(\tilde{w}_{1}, \tilde{w}_{2}, \ldots, \tilde{w}_{n}\right)^{T}=\left(\left\langle\tilde{w}_{1}^{\rho}, \tilde{w}_{1}^{\sigma}\right\rangle,\left\langle\tilde{w}_{2}^{\rho}, \tilde{w}_{2}^{\sigma}\right\rangle, \ldots\left\langle\tilde{w}_{n}^{\rho}, \tilde{w}_{n}^{\sigma}\right\rangle\right)^{T}$ and the optimal deviation variables $\tilde{d}_{i j}^{-}, \tilde{d}_{i j}^{+}, \tilde{e}_{i j}^{-}, \tilde{e}_{i j}^{+}$.

Theorem 7 Let $A^{k}=\left(a_{i j}^{k}\right)_{n \times n}=\left(\left\langle a_{i j, \rho}^{k}, a_{i j, \sigma}^{k}\right\rangle\right)_{n \times n}(k \in m)$ be a PFPR defined on alternative set $X=\left\{x_{1}, x_{2}, \ldots, x_{n}\right\}$, $A^{c}=\left(a_{i j, \rho}^{c}, a_{i j, \sigma}^{c}\right)_{n \times n}=\left(\sqrt{\prod_{k=1}^{m}\left(\left(a_{i j, \rho}^{k}\right)^{2}\right)^{\lambda^{k}}}, \sqrt{\prod_{k=1}^{m}\left(\left(a_{i j, \sigma}^{k}\right)^{2}\right)^{\lambda^{k}}}\right)_{n \times n}, i, j \in n$ be a comprehensive PFPR. Then, the inequality $C I\left(A^{c}\right) \leq \max _{1 \leq k \leq m}\left\{C I\left(A^{K}\right)\right\}$ holds.

Proof. From the aforementioned content, we have $d_{i j}^{c-}=\sum_{k=1}^{m} \lambda^{k} d_{i j}^{k-}, d_{i j}^{c+}=\sum_{k=1}^{m} \lambda^{k} d_{i j}^{k+}, e_{i j}^{c-}=\sum_{k=1}^{m} \lambda^{k} e_{i j}^{k-}$ and $e_{i j}^{c+}=\sum_{k=1}^{m} \lambda^{k} e_{i j}^{k+}$, where $d_{i j}^{k-}, d_{i j}^{k+}, e_{i j}^{k-}, e_{i j}^{k+}, i, j \in N, k \in M$ and $d_{i j}^{c-}, d_{i j}^{c+}, e_{i j}^{c-}, e_{i j}^{c+}, i, j \in N$ are the optimal deviation variables of the model (M-4.1) and model (M-4.2), respectively.
L e t $\tilde{A}^{k}=\left(\tilde{a}_{i j}^{k}\right)_{n \times n}=\left(\left\langle\tilde{a}_{i j, \rho}^{k}, \tilde{a}_{i j, \sigma}^{k}\right\rangle\right)_{n \times n}(k \in m)$, $\tilde{A}^{c}=\left(\tilde{a}_{i j}^{c}\right)_{n \times n}=\left(\left\langle\tilde{a}_{i j, \rho}^{c}, \tilde{a}_{i j, \sigma}^{c}\right\rangle\right)_{n \times n}, i, j \in n$, where

$\tilde{a}_{i j, \rho}^{k}=a_{i j, \rho}^{k} \cdot e^{\left(\tilde{d}_{i j}^{k-}-\tilde{d}_{i j}^{k+}\right)}, \quad \tilde{a}_{i j, \sigma}^{k}=a_{i j, \sigma}^{k} \cdot e^{\left(\tilde{e}_{i j}^{k-}-\tilde{e}_{i j}^{k+}\right)}$,

$\tilde{a}_{i j, \rho}^{c}=a_{i j, \rho}^{c} \cdot e^{\left(\tilde{d}_{i j}^{c-}-\tilde{d}_{i j}^{c+}\right)}, \quad \tilde{a}_{i j, \sigma}^{c}=a_{i j, \sigma}^{c} \cdot e^{\left(\tilde{e}_{i j}^{c-}-\tilde{e}_{i j}^{c+}\right)}$.

After deforming Eq. 4.8, we can obtain

$\ln \tilde{a}_{i j, \rho}^{k}-\ln a_{i j, \rho}^{k}=\tilde{d}_{i j}^{k-}-\tilde{d}_{i j}^{k+}$,

$\ln \tilde{a}_{i j, \sigma}^{k}-\ln a_{i j, \sigma}^{k}=\tilde{e}_{i j}^{k-}-\tilde{e}_{i j}^{k+}, \quad i, j \in N, \quad k \in M$,

$\ln \tilde{a}_{i j, \rho}^{c}-\ln a_{i j, \rho}^{c}=\tilde{d}_{i j}^{c-}-\tilde{d}_{i j}^{c+}$,

$\ln \tilde{a}_{i j, \sigma}^{c}-\ln a_{i j, \sigma}^{c}=\tilde{e}_{i j}^{c-}-\tilde{e}_{i j}^{c+}, \quad i, j \in N$.

Considering the above equation and $C I\left(A^{c}\right) \leq \overline{C I}$, the following process is derived:

$$
\begin{aligned}
C I\left(A^{c}\right) & =\frac{1}{n(n-1)} \sum_{\substack{i, j=1 \\
i<j}}^{n}\left(\left|\ln \left(\tilde{a}_{i j, \rho}^{c}\right)-\ln \left(a_{i j, \rho}^{c}\right)\right|+\left|\ln \left(\tilde{a}_{i j, \sigma}^{c}\right)-\ln \left(a_{i j, \sigma}^{c}\right)\right|\right) \\
& =\frac{1}{n(n-1)} \sum_{\substack{i, j=1 \\
i<j}}^{n}\left(\left|\tilde{d}_{i j}^{c-}-\tilde{d}_{i j}^{c+}\right|+\left|\tilde{e}_{i j}^{c-}-\tilde{e}_{i j}^{c+}\right|\right) \\
& =\frac{1}{n(n-1)} \sum_{\substack{i, j=1 \\
i<j}}^{n}\left(\left|\sum_{k=1}^{m} \lambda^{k} \tilde{d}_{i j}^{c-}-\sum_{k=1}^{m} \lambda^{k} \tilde{d}_{i j}^{c+}\right|+\left|\sum_{k=1}^{m} \lambda^{k} \tilde{e}_{i j}^{c-}-\sum_{k=1}^{m} \lambda^{k} \tilde{e}_{i j}^{c+}\right|\right) \\
& \leq \sum_{k=1}^{m} \lambda^{k}\left(\frac{1}{n(n-1)} \sum_{\substack{i j=1 \\
i<j}}^{n}\left(\left|\tilde{d}_{i j}^{c-}-\tilde{d}_{i j}^{c+}\right|+\left|\tilde{e}_{i j}^{c-}-\tilde{e}_{i j}^{c+}\right|\right)\right) \\
& =\sum_{k=1}^{m} \lambda^{k}\left(\frac{1}{n(n-1)} \sum_{\substack{i, j=1 \\
i<j}}^{n}\left(\left|\ln \left(\tilde{a}_{i j, \rho}^{k}\right)-\ln \left(a_{i j, \rho}^{k}\right)\right|+\left|\ln \left(\tilde{a}_{i j, \sigma}^{k}\right)-\ln \left(a_{i j, \sigma}^{k}\right)\right|\right)\right. \\
& =\sum_{k=1}^{m} \lambda^{k} \cdot C I\left(A^{k}\right) \leq \sum_{k=1}^{m} \lambda^{k} \cdot \max _{1 \leq k \leq m}\left\{C I\left(A^{k}\right)\right\}=\max _{1 \leq k \leq m}\left\{C I\left(A^{k}\right)\right\} .
\end{aligned}
$$


Overall, Theorem 7 holds, which completes the proof.

Inference 1 Let $A^{k}=\left(a_{i j}^{k}\right)_{n \times n}=\left(\left(a_{i j, \rho}^{k}, a_{i j, \sigma}^{k}\right)\right)_{n \times n}(k \in m)$ be a PFPR defined on alternative set $X=\left\{x_{1}, x_{2}, \ldots, x_{n}\right\}$, and $A^{c}=\left(a_{i j, \rho}^{c}, a_{i j, \sigma}^{c}\right)_{n \times n}=\left(\sqrt{\prod_{k=1}^{m}\left(\left(a_{i j, \rho}^{k}\right)^{2}\right)^{\lambda^{k}}}, \sqrt{\prod_{k=1}^{m}\left(\left(a_{i j, \sigma}^{k}\right)^{2}\right)^{\lambda^{k}}}\right)_{n \times n}, i, j \in$ be a comprehensive PFPR. If $C I\left(A^{k}\right) \leq \overline{C I}, k \in M$, then $C I\left(A^{c}\right) \leq \overline{C I}$ can be obtained, where $\overline{C I}$ is the given consistency index threshold.

Inference 2 Let $A^{k}=\left(a_{i j}^{k}\right)_{n \times n}=\left(\left(a_{i j, \rho}^{k}, a_{i j, \sigma}^{k}\right)\right)_{n \times n}(k \in m)$ be a PFPR defined on alternative set $X=\left\{x_{1}, x_{2}, \ldots, x_{n}\right\}$, and $A^{c}=\left(a_{i j, \rho}^{c}, a_{i j, \sigma}^{c}\right)_{n \times n}=\left(\sqrt{\prod_{k=1}^{m}\left(\left(a_{i j, \rho}^{k}\right)^{2}\right)^{\lambda^{k}}}, \sqrt{\prod_{k=1}^{m}\left(\left(a_{i j, \sigma}^{k}\right)^{2}\right)^{\lambda^{k}}}\right)_{n \times n}, i, j \in$ he a comprehensive PFPR. If $C I\left(A^{k}\right)=0, k \in M$, then $C I\left(A^{c}\right)=0$ can be obtained, which shows that if all PFPRs $A^{k}$ are multiplicative consistent, then the comprehensive PFPR $A^{c}$ is also multiplicatively consistent.

\section{Consensus reaching algorithm}

To improve the consensus of all decision-makers in the decision-making problem, this section designs an algorithm to improve the consensus level, which is realized by filtering the preference relation with the worst consensus and adjusting it to reach the consensus index threshold.

\section{$(\mathrm{A}-4.1)$}

Input: A set of PFPRs $A^{1}, A^{2}, \ldots, A^{m}$, a group consensus index threshold $\overline{G C D}$, and parameter $\theta(0<\theta<1)$.

Output: Adjusted PFPR $A^{k *}(k \in M)$, the degree of group consensus GCD*, and iterations $l$.

Step 1: Let $A^{k(l)}=\left(a_{i j}^{k(l)}\right)_{n \times n}=A^{k}=\left(a_{i j}^{k}\right)_{n \times n}, k \in M, l=0$.
Step 2: The degrees of individual consensus $\operatorname{ICD}^{(l)}$ and degrees of group consensus $\mathrm{GCD}^{(l)}$ of PFPRs $A^{k(l)}(k \in M)$ are calculated by Eq. 4.1 and Eq. 4.2, respectively.

Step 3: Check for a consensus for PFPRs. If $\mathrm{GCD}^{(l)} \leq \overline{\mathrm{GCD}}$, we continue to step 5. Otherwise, go to step 4.

Step 4: Focus on the PFPR with the worst consensus and adjust it.

Assume that $\operatorname{ICD}\left(A^{\varphi(l)}\right)=\max _{k}\left\{\operatorname{ICD}\left(A^{k(l)}\right)\right\}$, and then $A^{\varphi(l)}$ is called the worst consensus PFPR. Let $A^{k(l+1)}=\left(a_{i j}^{k(l+1)}\right)_{n \times n}=\left(a_{i j, \rho}^{k(l+1)}, a_{i j, \sigma}^{k(l+1)}\right)_{n \times n}$, which satisfies

$a_{i j, \rho}^{k(l+1)}= \begin{cases}\left(a_{i j, \rho}^{k(l)}\right)^{\theta}\left(\prod_{k \neq \varphi} a_{i j, \rho}^{k(l)}\right)^{\frac{1-\theta}{m-1}}, & k=\varphi, \\ a_{i j, \rho}^{k(l)}, & k \neq \varphi,\end{cases}$

$a_{i j, \sigma}^{k(l+1)}= \begin{cases}\left(a_{i j, \sigma}^{k(l)}\right)^{\theta}\left(\prod_{k \neq \varphi} a_{i j, \sigma}^{k(l)}\right)^{\frac{1-\theta}{m-1}}, & k=\varphi, \\ a_{i j, \sigma}^{k(l)}, & k \neq \varphi .\end{cases}$

Then, let $l=l+1$, return to step 2 .

Step 5: Let the iterative PFPR $A^{k *}=A^{k(l)}(k \in M)$ and $\mathrm{GCD}^{*}=\mathrm{GCD}^{(l)}$. The output adjusted PFPR $A^{k *}(k \in M)$, the degree of group consensus GCD*, and iterations $l$.

Step 6: End.

\section{Algorithm for group decision-making with PFPRs}

This section will integrate the contents of the third and fourth sections to build a decision support model based on consistency and consensus, which can ensure that all PFPRs reach the preset consensus level under the premise of satisfying the acceptable multiplicative consistency. In addition, the model is used to calculate the standardized weight vector
Table 1 Values of score function and ranking results of different methods

\begin{tabular}{lll}
\hline Methods & Values of score function & Ranking results \\
\hline Chu et al. [18] & $(-0.0910,-0.2979,-0.6574,-0.8640)$ & $x_{1}^{\text {Chu }}>x_{2}^{\text {Chu }}>x_{3}^{\text {Chu }}>x_{4}^{\text {Chu }}$ \\
Jin et al. [19] & $(-0.1184,-0.5200,-0.5916,-0.7700)$ & $x_{1}^{\text {Jin }}>x_{2}^{\text {Jin }}>x_{3}^{\text {Jin }}>x_{4}^{\text {Jin }}$ \\
Lin and Wang [20] & $(-0.1776,-0.4090,-0.6590,-0.7544)$ & $x_{1}^{\text {Lin }}>x_{2}^{\text {Lin }}>x_{3}^{\text {Lin }}>x_{4}^{\text {Lin }}$ \\
Yang et al. [35] & $(-0.1543,-0.2684,-0.6548,-0.7944)$ & $x_{1}^{\text {Yang }}>x_{2}^{\text {Yang }}>x_{3}^{\text {Yang }}>x_{4}^{\text {Yang }}$ \\
Mandal and Ranadive & $(0.6072,0.3703,0.2519,0.2054)$ & $x_{1}^{\text {Mandal }}>x_{2}^{\text {Mandal }}>x_{3}^{\text {Mandal }}>x_{4}^{\text {Mandal }}$ \\
[34] & $(-0.4832,-0.3602,-0.7061,-0.6543)$ & $x_{2}^{\text {He }}>x_{1}^{\text {He }}>x_{4}^{\text {He }}>x_{3}^{\text {He }}$ \\
He et al. [40] & $(-0.0747,-0.4753,-0.6741,-0.7762)$ & $x_{1}>x_{2}>x_{3}>x_{4}$ \\
Our method & &
\end{tabular}


of the comprehensive PFPRs. Finally the most reasonable and reliable decision results are obtained.

For the convenience of comprehension, we give the process flowchart and text descriptions of the decision support model with PFPRs as follows.

\section{$(\mathbf{A}-5.1)$}

Input: A set of PFPRs $A^{1}, A^{2}, \ldots, A^{m}$ supported by DMs.

Output: The best alternative $x^{\Delta}$.

\section{Stage 1: Check order consistency}

If the PFPRs satisfy the constraints in Definition 7, then they are order consistent and the ranking results can be directly output. Otherwise, go to the next stage.

\section{Stage 2: Consistency control process}

The improved consistency algorithm (A-3.2) is used to adjust and obtain the PFPRs $A^{1}, A^{2}, \ldots, A^{m}$ with acceptable multiplicative consistency.

\section{Stage 3: Consensus building process}

The adjusted PFPRs $A^{1 *}, A^{2 *}, \ldots, A^{m *}$ of acceptable multiplicative consistency are calculated by the consensus improving algorithm (A-4.1), so that the degree of group consensus reaches the consensus index threshold.

\section{Stage 4: Alternatives selection process}

Equation (30) is used to aggregate the PFPRs $A^{1 \Delta}, A^{2 \Delta}, \ldots, A^{m \Delta}$ into comprehensive PFPR $A^{c \Delta}$. According to the ranking of the standardized weight vector calculated by the optimization model (M-4.2), the alternatives are sorted to select the best one $x^{\Delta}$ (Fig. 1)

\section{Numerical example and comparison analysis}

In this section, we use a numerical example to illustrate the proposed GDM method and compare the experimental results of the proposed GDM method with those of the GDM methods.

\section{Numerical example}

In recent years, the rapid development of the economy has brought opportunities to various industries, but has also brought many challenges. For example, increasingly more enterprises are facing financial difficulties, which makes it important to establish an effective financial distress early warning model to prevent financial crises and risk management.

To achieve a better foothold and long-term development, a listed company establishes a perfect financial early warning system. Through comprehensive analysis, it is found that the main indicators that affect the financial situation of the enterprise are the following: solvency $x_{1}$, operations $x_{2}$, profitability $x_{3}$, and development $x_{4}$. To explore the importance of these four indicators to the financial early warning system, the listed company now invites three experts in related fields to evaluate and analyze the above four impact indicators systematically. The weight vector of the experts is $\lambda=(0.3,0.4,0.3)^{T}$. Besides, to simplify the decision-making process, preference relations are useful tools that only require the DMs to judge two alternatives at a time. Furthermore, PFVs are flexible in expressing the DMs' hesitancy, where there is more than one value for judgments. Considering these two aspects, PFPRs are used for this GDM problem.

Therefore, experts use PFNs to evaluate the above four impact indicators according to professional knowledge and financial experience. The final PFPRs are as follows:

$$
\begin{aligned}
A^{1} & =\left[\begin{array}{llll}
(\sqrt{2} / 2, \sqrt{2} / 2) & (0.60,0.70) & (0.70,0.60) & (0.65,0.70) \\
(0.70,0.60) & (\sqrt{2} / 2, \sqrt{2} / 2) & (0.80,0.30) & (0.75,0.45) \\
(0.60,0.70) & (0.30,0.80) & (\sqrt{2} / 2, \sqrt{2} / 2) & (0.75,0.60) \\
(0.70,0.65) & (0.45,0.75) & (0.60,0.75) & (\sqrt{2} / 2, \sqrt{2} / 2)
\end{array}\right], A^{2}=\left[\begin{array}{llll}
(\sqrt{2} / 2, \sqrt{2} / 2) & (0.75,0.55) & (0.80,0.50) & (0.80,0.50) \\
(0.55,0.75) & (\sqrt{2} / 2, \sqrt{2} / 2) & (0.60,0.70) & (0.80,0.55) \\
(0.50,0.80) & (0.70,0.60) & (\sqrt{2} / 2, \sqrt{2} / 2) & (0.75,0.55) \\
(0.50,0.80) & (0.55,0.80) & (0.55,0.75) & (\sqrt{2} / 2, \sqrt{2} / 2)
\end{array}\right] \\
A^{3} & =\left[\begin{array}{llll}
(\sqrt{2} / 2, \sqrt{2} / 2) & (0.75,0.55) & (0.75,0.60) & (0.80,0.35) \\
(0.55,0.75) & (\sqrt{2} / 2, \sqrt{2} / 2) & (0.65,0.45) & (0.40,0.45) \\
(0.60,0.75) & (0.45,0.65) & (\sqrt{2} / 2, \sqrt{2} / 2) & (0.40,0.65) \\
(0.35,0.80) & (0.45,0.40) & (0.65,0.40) & (\sqrt{2} / 2, \sqrt{2} / 2)
\end{array}\right]
\end{aligned}
$$

Some preset parameters are given as follows: consistency index threshold level $\overline{C I}=0.1$, group consistency index threshold level $\overline{\mathrm{GCD}}=0.1$, consistency adjustment

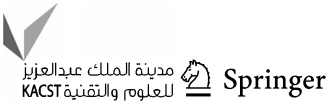


Table 2 Correlations of different methods

\begin{tabular}{llllllll}
\hline & & Chu [18] & Jin [19] & Lin [20] & Yang [35] & Mandal [34] & Our method \\
\hline Chu [18] & Pearson correlation & 1 & 0.921 & $0.988^{*}$ & $0.994^{* *}$ & 0.942 & 0.949 \\
& Sig. (2-tailed) & & 0.079 & 0.012 & 0.006 & 0.058 & 0.051 \\
Jin [19] & Pearson correlation & 0.921 & 1 & $0.954^{*}$ & 0.876 & $0.983^{*}$ & $0.986^{*}$ \\
& Sig. (2-tailed) & 0.079 & & 0.046 & 0.124 & 0.017 & 0.014 \\
Lin [20] & Pearson correlation & $0.988^{*}$ & $0.954^{*}$ & 1 & $0.976^{*}$ & $0.980^{*}$ & $0.983^{*}$ \\
& Sig. (2-tailed) & 0.012 & 0.046 & & 0.024 & 0.020 & 0.017 \\
Yang [35] & Pearson correlation & $0.994^{* *}$ & 0.876 & $0.976^{*}$ & 1 & 0.913 & 0.920 \\
& Sig. (2-tailed) & 0.006 & 0.124 & 0.024 & & 0.087 & 0.080 \\
\multirow{2}{*}{ Mandal [34] } & Pearson correlation & 0.942 & $0.983^{*}$ & $0.980^{*}$ & 0.913 & 1 & $0.996^{* *}$ \\
& Sig. (2-tailed) & 0.058 & 0.017 & 0.020 & 0.087 & & 0.004 \\
Our method & Pearson correlation & 0.949 & $0.986^{*}$ & $0.983^{*}$ & 0.920 & $0.996^{* *}$ & 1 \\
& Sig. (2-tailed) & 0.051 & 0.014 & 0.017 & 0.080 & 0.004 & \\
\hline
\end{tabular}

*Correlation is significant at the 0.05 level (2-tailed)

**Correlation is significant at the 0.01 level (2-tailed) parameter $\gamma=0.2$, and consistency adjustment parameter $\theta=0.3$. The detailed stages on the latter are shown in "Appendix".

As international competition intensifies and the market environment changes, enterprises will inevitably suffer from various financial crises. Establishing a financial early warning system is conducive to preventing and overcoming financial crises. To guard against financial risks, the listed company prefers to establish an effective financial early warning system based on several indicators affecting its financial situation.

From the stages in "Appendix", we obtain $s\left(x_{1}\right)=-0.0747, s\left(x_{2}\right)=-0.4753, s\left(x_{3}\right)=-0.6741$, and $s\left(x_{4}\right)=-0.7762$ via Definition 5. Then, the normalized Pythagorean fuzzy weight vector is ranked $\tilde{w}_{1}>\tilde{w}_{2}>\tilde{w}_{3}>\tilde{w}_{4}$. Therefore, the rank results of importance of the influencing factors are solvency $>$ operations $>$ profitability $>$ development $\left(x_{1}>x_{2}>x_{3}>x_{4}\right)$, indicating that the most important interfering factor is solvency $x_{1}$. Moreover, the comprehensive PFPR $A^{c \Delta}$ also shows that the preferred factor $x_{1}$ is higher than the other three factors by the three experts $x_{j}(j=2,3,4)$, such as $a_{12}^{c \Delta}=(0.7299,0.5672), a_{13}^{c \Delta}=(0.7784,0.5129)$ and $a_{14}^{c \Delta}=(0.7762,0.4833)$. Furthermore, most of the raw information in the PFPRs given by the three experts is robust enough to reflect that the preference value of $x_{1}$ is superior to those of the others. E.g., $a_{12}^{2}=(0.75,0.55)$ represents the degree of preference of solvency $x_{1}$ relative to operations $x_{2}$. Obviously, this is consistent with the results obtained in this paper, which side by side proves that the method of this paper is robust and reasonable.

Therefore, under the analysis and judgment of the three experts in the related field, it is concluded that solvency, operations, profitability, and development are all important indicators reflecting financial status; however, to avoid risks effectively, listed companies should give primary consideration to their own solvency when establishing a financial early warning system.

Generally, solvency is the ability to pay debts as they fall due. If there is a problem with solvency, it will definitely pose a threat to the entire financial situation of an enterprise, and may even cause the enterprise to experience a financial crisis. Therefore, in the fierce market competition, the first goal of an enterprise to survive is to cover its expenses with revenues and pay off its debts on time.

\section{Comparison analysis}

The following is the comparison and analysis of methods of [18-20, 34, 35, 39], which includes the following main aspects.

1. To highlight the excellent properties of multiplicative consistency, we will compare multiplicative consistency with the method of additive consistency. Therefore, the methods proposed by Chu et al. [18] and Yang et al. [35] are used to find the optimal solution to the above problem.

2. To determine whether the decision-making information of the Pythagorean fuzzy environment is more comprehensive than that of the intuitionistic fuzzy environment, we will compare the proposal with the IFPR methods proposed by Chu et al. [18], Jin et al. [19] and Lin and Wang [20].

3. To accentuate the consensus in GDM, we will compare the proposal with the individual decision-making approach by He et al. [39] and the approach that consid- 


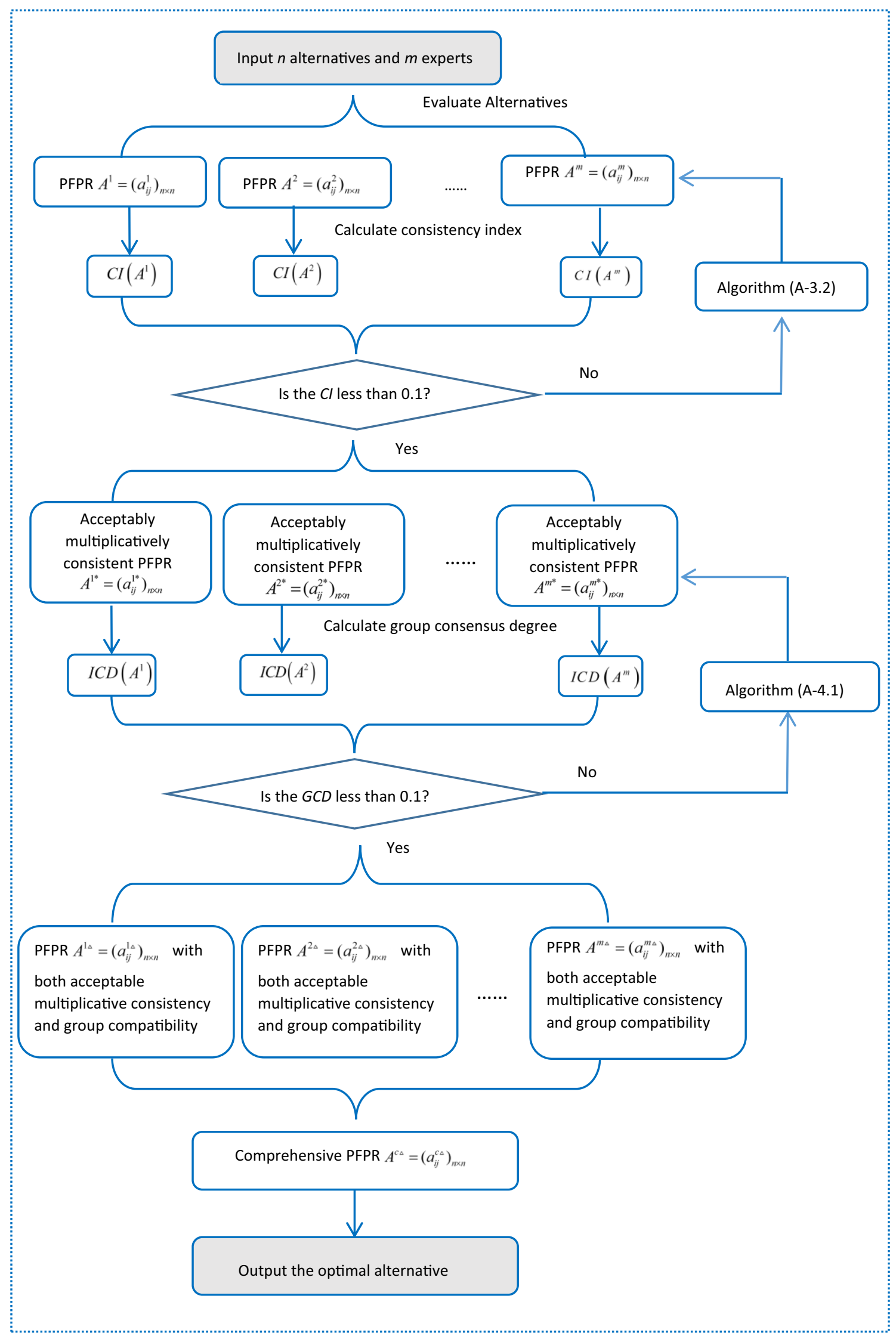

Fig. 1 Process flowchart of decision support model with PFPRs 
ers only consistency proposed by Jin et al. [19], Mandal and Ranadive [34], and Lin and Wang [20].

Considering the relationship between PFPRs and IFPRs, we will compare the methods [18-20] under an intuitionistic fuzzy environment to highlight the advantages of PFPRs. First, according to the transformation between them, the following IFPRs are obtained: to operations $x_{2}$ is 0.525 , and the degree of certainty that solvency $x_{1}$ is non-preferred to operations $x_{2}$ is 0.45 . Obviously, solvency $x_{1}$ cannot be inferior to operation $x_{2}$. Similarly, profitability $x_{3}$ should not be inferior to development $x_{4}$ by $A_{34}^{\odot}=(0.4750,0.4500)$. Therefore, our method is more robust than that of [39].

Second, from the four indicator rankings obtained by our method with those of Chu et al. [18], Jin et al. [19], Lin

$$
\begin{aligned}
& A^{1}=\left[\begin{array}{llll}
(0.5000,0.5000) & (0.3600,0.4900) & (0.4900,0.3600) & (0.4225,0.4900) \\
(0.4900,0.3600) & (0.5000,0.5000) & (0.6400,0.0900) & (0.5625,0.2025) \\
(0.3600,0.4900) & (0.0900,0.6400) & (0.5000,0.5000) & (0.5625,0.3600) \\
(0.4900,0.4225) & (0.2025,0.5625) & (0.3600,0.5625) & (0.5000,0.5000)
\end{array}\right], \\
& A^{2}=\left[\begin{array}{llll}
(0.5000,0.5000) & (0.5625,0.3025) & (0.6400,0.2500) & (0.6400,0.2500) \\
(0.3025,0.5625) & (0.5000,0.5000) & (0.3600,0.4900) & (0.6400,0.3025) \\
(0.2500,0.6400) & (0.4900,0.3600) & (0.5000,0.5000) & (0.5625,0.3025) \\
(0.2500,0.6400) & (0.3025,0.6400) & (0.3025,0.5625) & (0.5000,0.5000)
\end{array}\right], \\
& A^{3}=\left[\begin{array}{llll}
(0.5000,0.5000) & (0.5625,0.3025) & (0.5625,0.3600) & (0.6400,0.1225) \\
(0.3025,0.5625) & (0.5000,0.5000) & (0.4225,0.2025) & (0.1600,0.2025) \\
(0.3600,0.5625) & (0.2025,0.4225) & (0.5000,0.5000) & (0.1600,0.4225) \\
(0.1225,0.6400) & (0.2025,0.1600) & (0.4225,0.1600) & (0.5000,0.5000)
\end{array}\right] .
\end{aligned}
$$

Considering that the method proposed by He et al. [39] solves an individual decision-making problem, the original PFPRs need to be converted to an overall decision-making matrix. The overall PFPR $A^{\odot}$ can be obtained by utilizing the method in [37]. We need to convert the above PFPRs $A^{k}=\left(a_{i j}^{k}\right)_{4 \times 4}(k=1,2,3)$ of these four factors for the financial early warning system into the following overall PFPRs $A^{\odot}$ :

$A^{\odot}=\left[\begin{array}{cccc}(\sqrt{2} / 2, \sqrt{2} / 2) & (0.5250,0.4500) & (0.5625,0.4250) & (0.5625,0.3875) \\ (0.4500,0.5250) & (\sqrt{2} / 2, \sqrt{2} / 2) & (0.5125,0.3625) & (0.4875,0.3625) \\ (0.4250,0.5625) & (0.3625,0.5125) & (\sqrt{2} / 2, \sqrt{2} / 2) & (0.4750,0.4500) \\ (0.3875,0.5625) & (0.3625,0.4875) & (0.4500,0.4750) & (\sqrt{2} / 2, \sqrt{2} / 2)\end{array}\right]$. and Wang [20], Yang et al. [35], and Mandal and Ranadive [34], we can see that the ranking results are consistent, i.e., solvency $>$ operations $>$ profitability $>$ development $\left(x_{1}>x_{2}>x_{3}>x_{4}\right)$, but with some differences in approaches.

1. Compared with the methods proposed by Chu et al. [18], Yang et al. [35] found the optimal solution to the above problem. We know that when additive consistency is used to describe the incomplete information in

Based on the example of financial crisis and risk management, the calculation results of the above five methods are shown in Table 1. To make the analysis not too redundant and to better highlight the results, the specific calculation procedure is omitted.

First, compared with the method proposed by He et al. [39], we find that the results obtained by the method in [39] are different from those obtained by our proposed method. The method in [39] yields a ranking result of $x_{2}^{\mathrm{He}}>x_{1}^{\mathrm{He}}>x_{4}^{\mathrm{He}}>x_{3}^{\mathrm{He}}$, while our method produces a ranking result of $x_{1}>x_{2}>x_{3}>x_{4}$. However, from the overall PFPR matrix $A^{\odot}$, we have $A_{12}^{\odot}=(0.5250,0.4500)$, which means the degree of certainty that solvency $x_{1}$ is preferred
FPRs, an overflow range of 0 to 1 may be generated. Compared with additive consistency [18, 35], multiplicative consistency does not have information spillover in FPRs, or PFPRs. Therefore, the advantages of our decision support model are obvious. In response to PFs that do not achieve acceptable consistency, Chu et al. [18] built non-preferred relations for adjustment. This method can consider the additive consistency of the nonpreferred relations, but the process is complicated and information is easily lost. Our decision support model is directly based on the decision-making information given by experts, making full use of the original decision-making information for decision-making, which can reduce 
the information loss in the decision-making process and increase the reliability of the final results. By comparison, our method is simpler and more convenient.

2. As above, the decision results by Chu et al. [18], Jin et al. [19] and Lin and Wang [20] are also consistent with our final result, which again proves the excellent performance of the decision support model proposed in this paper. Although Jin et al. [19] constructed an improved model of the multiplicative consistency of IFPRs, our integrated environment is based on PFPRs, which is more extensive and sweeping than IFPRs in describing decision information. Moreover, the group decision model based on multiple IFPRs is just a simple extension of the decision model based on individual IFPRs. The model does not consider the consistency and compatibility of IFPRs, but our group decision model is the opposite. In our decision support model, we not only discuss how to improve the consistency of PFPRs, but also consider how to enhance the compatibility between them. Therefore, the virtues of this method are self-evident.

3. We compare the proposed method with the individual decision-making approach by $\mathrm{He}$ et al. [39] and the approach that considers only consistency proposed by Jin et al. [19], Mandal and Ranadive [34], and Lin and Wang [20]. He et al. [39] focused on multiplicatively consistent PFPRs, which is similar to this paper. Their method only considered the individual decision problem, while our method discusses the GDM consensus problem. Although Jin et al. [19] considered GDM, GDM was only an aggregation of individual decisions and did not consider the problem of non-consensus opinions among experts in GDM. As for Mandal and Ranadive [34], a consensus relationship was constructed. However, there is a problem with the definition of the relevant equation, which makes the value on the diagonal of the constructed consensus relation not equal to one. Thus, our method is more practical.

Through the introduction of the above different methods of [18-20,34, 35], it is found that the ranking results of the impact of factors are consistent (see Table 1), which shows that the decision support model constructed in this paper is reasonable and effective. Furthermore, the decision support model proposed in this paper integrates a variety of algorithm models such as a consistency improving algorithm and a consensus building algorithm. Therefore, our research methods consider decision information from multiple perspectives and solve decision-making problems at different levels. Besides, there is no information overflow in multiplicative consistency, which is more suitable for PFPRs than additive consistency. However, the degree of spatial membership of intuitionistic fuzzy environment is less than that of Pythagorean fuzzy environment, which is equivalent to the IFNs must be the PFNs. Otherwise, this does not hold. Therefore, the PFPRs can solve the problem that IFPRs are incompetent. In conclusion, it is valuable to build a decision support model with PFPRs based on multiplicative consistency.

To make a comparison, we count the Pearson correlations of rankings similarity via SPSS, as shown in Table 2.

According to the data of correlations of different methods with the same ranking, there are some correlations among these methods, and the difference is only the strength of the correlations. The correlation coefficients of our method and methods in [19] and [20] are less than 0.05, and are significantly correlated. In particular, the correlation coefficient between our method and the method in [34] is $0.006<1$, indicating a strong correlation between them.

\section{Conclusions}

Considering that different fuzzy environments, consistency improvement, consensus reaching, and the method of ranking weights are the key research topics, this paper focuses on the preference relations in a Pythagorean fuzzy environment.

This paper provides several significant contributions to group decision-making with PFPRs as follows:

1. A novel concept of multiplicatively consistent PFPRs is introduced. The advantage of the proposed multiplicative consistency is that it can overcome the malpractice of additive consistency with PFPRs.

2. A consistency measure index of PFPRs is introduced. Moreover, when the CI does not reach the expected threshold, a consistency improving model is designed to achieve the expected threshold.

3. The ICD and GCD are developed to measure the consensus level. If the GCD is not within the expected threshold, the consensus reaching model is modified to reach the expected threshold.

4. For the individual decision-making problem, order consistency is defined to evaluate it efficiently. Furthermore, a decision support model, which is based on a consistency improving model and consensus reaching model, is constructed for the GDM problem.

However, we do not conduct deep discussions on how the iteration parameters will affect the iterative speed and final decision results, which will be considered in the future. In addition, the weight of the experts in this paper is fixed, without considering the complexity of practical problems. The acquisition of incomplete weight information will be included in the following research. 


\section{Appendix}

Continued from the numerical example above.

Stage 1: Judge whether the PFPRs $A^{k}=\left(a_{i j}^{k}\right)_{4 \times 4}(k=1,2,3)$ are order consistent. From the analysis of the PFPRs $A^{k}=\left(a_{i j}^{k}\right)_{4 \times 4}(k=1,2,3)$, it can be seen that the PFPRs do not conform to constraints in Definition 7. Thus, they are not order consistent, and we proceed to the next stage.

\section{Stage 2: Consistency control process}

In this stage, the acceptable multiplicative consistency of the three PFPRs $A^{k}=\left(a_{i j}^{k}\right)_{4 \times 4}(k=1,2,3)$ is tested.

First, multiplicatively consistent PFPRs can be obtained as follows:

$$
\begin{array}{r}
\hat{A}^{1}=\left[\begin{array}{llll}
(\sqrt{2} / 2, \sqrt{2} / 2) & (0.6000,0.7428) & (0.6631,0.6000) & (0.6563,0.5881) \\
(0.7428,0.6000) & (\sqrt{2} / 2, \sqrt{2} / 2) & (0.7577,0.5539) & (0.7500,0.5430) \\
(0.6000,0.6631) & (0.5539,0.7577) & (\sqrt{2} / 2, \sqrt{2} / 2) & (0.6059,0.6000) \\
(0.5881,0.6563) & (0.5430,0.7500) & (0.6000,0.6059) & (\sqrt{2} / 2, \sqrt{2} / 2)
\end{array}\right], \\
\hat{A}^{2}=\left[\begin{array}{llll}
(\sqrt{2} / 2, \sqrt{2} / 2) & (0.7867,0.5500) & (0.7694,0.6010) & (0.8000,0.5000) \\
(0.5500,0.7867) & (\sqrt{2} / 2, \sqrt{2} / 2) & (0.6000,0.6704) & (0.6239,0.5578) \\
(0.6010,0.7694) & (0.6704,0.6000) & (\sqrt{2} / 2, \sqrt{2} / 2) & (0.6817,0.5455) \\
(0.5000,0.8000) & (0.5578,0.6239) & (0.5455,0.6817) & (\sqrt{2} / 2, \sqrt{2} / 2)
\end{array}\right], \\
\hat{A}^{3}=\left[\begin{array}{llll}
(\sqrt{2} / 2, \sqrt{2} / 2) & (0.7500,0.5500) & (0.7972,0.4047) & (0.7515,0.4047) \\
(0.5500,0.7500) & (\sqrt{2} / 2, \sqrt{2} / 2) & (0.6500,0.4500) & (0.6128,0.4500) \\
(0.4047,0.7972) & (0.4500,0.6500) & (\sqrt{2} / 2, \sqrt{2} / 2) & (0.4509,0.4783) \\
(0.4047,0.7515) & (0.4500,0.6128) & (0.4783,0.4509) & (\sqrt{2} / 2, \sqrt{2} / 2)
\end{array}\right] .
\end{array}
$$

$$
\begin{array}{r}
A^{1 \prime}=\left[\begin{array}{llll}
(\sqrt{2} / 2, \sqrt{2} / 2) & (0.6000,0.7084) & (0.6925,0.6000) & (0.6513,0.6760) \\
(0.7084,0.6000) & (\sqrt{2} / 2, \sqrt{2} / 2) & (0.7914,0.3391) & (0.7500,0.4672) \\
(0.6000,0.6925) & (0.3391,0.7914) & (\sqrt{2} / 2, \sqrt{2} / 2) & (0.7187,0.6000) \\
(0.6760,0.6513) & (0.4672,0.7500) & (0.6000,0.7187) & (\sqrt{2} / 2, \sqrt{2} / 2)
\end{array}\right], \\
A^{3 \prime}=\left[\begin{array}{llll}
(\sqrt{2} / 2, \sqrt{2} / 2) & (0.7500,0.5500) & (0.7592,0.5546) & (0.7901,0.3603) \\
(0.5500,0.7500) & (\sqrt{2} / 2, \sqrt{2} / 2) & (0.6500,0.4500) & (0.4356,0.4500) \\
(0.5546,0.7592) & (0.4500,0.6500) & (\sqrt{2} / 2, \sqrt{2} / 2) & (0.4097,0.6113) \\
(0.3603,0.7901) & (0.4500,0.4356) & (0.6113,0.4097) & (\sqrt{2} / 2, \sqrt{2} / 2)
\end{array}\right],
\end{array}
$$

Next, we will calculate the consistency indexes $C I\left(A^{k}\right)(k=1,2,3)$ corresponding to the PFPRs.

$C I\left(A^{1}\right)=0.1138, C I\left(A^{2}\right)=0.0567$, and $C I\left(A^{3}\right)=0.1263$.

Compared with the consistency index threshold $\overline{C I}=0.1$, it is easy to find that $C I\left(A^{1}\right)>\overline{C I}, C I\left(A^{2}\right)<\overline{C I}$, and $C I\left(A^{3}\right)>\overline{C I}$, which means that the PFPRs $A^{1}=\left(a_{i j}^{1}\right)_{4 \times 4}$ and $A^{3}=\left(a_{i j}^{3}\right)_{4 \times 4}$ are not acceptably multiplicatively consistent. Therefore, they should be adjusted via the consistency improving algorithm. Then, we have the following adjusted PFPRs $A^{1 \prime}=\left(a_{i j}^{1 \prime}\right)_{4 \times 4}$ and $A^{3 \prime}=\left(a_{i j}^{3 \prime}\right)_{4 \times 4}$ : 
whose consistent indexes are $C I\left(A^{1 \prime}\right)=0.0911<\overline{C I}$ and $C I\left(A^{3 \prime}\right)=0.1011>\overline{C I}$, respectively. Therefore, $A^{3 \prime}$ is adjusted to meet the condition that its consistency index is less than 0.1. Then, let $A^{3 \prime}=A^{3 \prime \prime}$. One can obtain

$A^{3^{\prime \prime}}=\left[\begin{array}{lll}(\sqrt{2} / 2, \sqrt{2} / 2) & (0.7500,0.5500) & (0.7667,0.5072) \\ (0.5500,0.7500) & (\sqrt{2} / 2, \sqrt{2} / 2) & (0.6500,0.4500) \\ (0.5072,0.7667) & (0.4500,0.6500) & (\sqrt{2} / 2, \sqrt{2} / 2) \\ (0.3688,0.7822) & (0.4500,0.4664) & (0.5820,0.4176)\end{array}\right.$
Based on the adjusted PFPRs $A^{1 *}, A^{2 *}, \ldots, A^{m *}$ with acceptable multiplicative consistency, we will check their consensus.

First, let $l=0$, and $A^{k *(l)}=A^{k *}=\left(a_{i j}^{k *}\right)_{4 \times 4}, k=1,2,3$. The with $C I\left(A^{3 \prime \prime}\right)=0.0786<\overline{C I}$. Through this previous process of improving the consistency, all PFPRs are acceptably multiplicatively consistent. Then, let the PFPRs $A^{1 *}=A^{1 \prime}, A^{2 *}=A^{2}$ and $A^{3 *}=A^{3 \prime \prime}$, and proceed to the next stage 3 .

\section{Stage 3: Consensus building process}

$\begin{aligned} A^{1 *} & =\left[\begin{array}{llll}(\sqrt{2} / 2, \sqrt{2} / 2) & (0.6000,0.7084) & (0.6925,0.6000) & (0.6513,0.6760) \\ (0.7084,0.6000) & (\sqrt{2} / 2, \sqrt{2} / 2) & (0.7914,0.3391) & (0.7500,0.4672) \\ (0.6000,0.6925) & (0.3391,0.7914) & (\sqrt{2} / 2, \sqrt{2} / 2) & (0.7187,0.6000) \\ (0.6760,0.6513) & (0.4672,0.7500) & (0.6000,0.7187) & (\sqrt{2} / 2, \sqrt{2} / 2)\end{array}\right], \\ A^{2 *} & =\left[\begin{array}{llll}(\sqrt{2} / 2, \sqrt{2} / 2) & (0.7500,0.5500) & (0.8000,0.5000) & (0.8000,0.5000) \\ (0.5500,0.7500) & (\sqrt{2} / 2, \sqrt{2} / 2) & (0.6000,0.7000) & (0.8000,0.5500) \\ (0.5000,0.8000) & (0.7000,0.6000) & (\sqrt{2} / 2, \sqrt{2} / 2) & (0.7500,0.5500) \\ (0.5000,0.8000) & (0.5500,0.8000) & (0.5500,0.7500) & (\sqrt{2} / 2, \sqrt{2} / 2)\end{array}\right], \\ A^{3 *}= & {\left[\begin{array}{llll}(\sqrt{2} / 2, \sqrt{2} / 2) & (0.7500,0.5500) & (0.7667,0.5072) & (0.7822,0.3688) \\ (0.5500,0.7500) & (\sqrt{2} / 2, \sqrt{2} / 2) & (0.6500,0.4500) & (0.4664,0.4500) \\ (0.4047,0.7972) & (0.4500,0.6500) & (\sqrt{2} / 2, \sqrt{2} / 2) & (0.4176,0.5820) \\ (0.4047,0.7515) & (0.4500,0.6128) & (0.4783,0.4509) & (\sqrt{2} / 2, \sqrt{2} / 2)\end{array}\right] . }\end{aligned}$ degree of individual consensus ICD and the degree of group consensus GCD with PFPRs are calculated as follows:

$\operatorname{ICD}\left(A^{1 *(0)}\right)=0.2404, \operatorname{ICD}\left(A^{2 *(0)}\right)=0.2065, \operatorname{ICD}\left(A^{3 *(0)}\right)$

$$
=0.2245 \text { and } \mathrm{GCD}=0.2238 \text {. }
$$

Du e to $\quad$ GCD $=0.2238>\overline{\mathrm{GCD}}=0.1, \quad \mathrm{it}$ is necessary to adjust the worst PFPR in the 
consensus. From the above operation results, we know $\operatorname{ICD}\left(A^{1 *(0)}\right)=\max _{1 \leq k \leq 3}\left\{\operatorname{ICD}\left(A^{k *(0)}\right)\right\}$. Then, the following $A^{1 *(1)}$ is adjusted by Eq. (35):

$A^{1 *(1)}=\left[\begin{array}{llll}(\sqrt{2} / 2, \sqrt{2} / 2) & (0.7014,0.5934) & (0.7548,0.5308) & (0.7462,0.4920) \\ (0.5934,0.7014) & (\sqrt{2} / 2, \sqrt{2} / 2) & (0.6705,0.4825) & (0.6496,0.4882) \\ (0.5629,0.7490) & (0.4825,0.6705) & (\sqrt{2} / 2, \sqrt{2} / 2) & (0.6033,0.5758) \\ (0.5056,0.7521) & (0.4882,0.6882) & (0.5985,0.5942) & (\sqrt{2} / 2, \sqrt{2} / 2)\end{array}\right]$.
As stated above, $\mathrm{GCD}=0.0868<\overline{\mathrm{GCD}}$ which means that the group consensus degree of the adjusted PFPRs reaches the pre standard.
In addition, let $A^{2 *(1)}=A^{2 *(0)}$ and $A^{3 *(1)}=A^{3 *(0)}$. Next, the ICDs and GCD of the iterated PFPRs are calculated separately as follows:
Finally, let $\quad A^{k \Delta}=A^{k *(1)}, k=1,2,3, \quad$ and $\mathrm{GCD}^{\Delta}=\mathrm{GCD}=0.0868$. We output PFPRs $A^{k \Delta}, k=1,2,3$. They have an acceptable multiplicative consistency and

$\operatorname{ICD}\left(A^{1 *(1)}\right)=0.1188, \operatorname{ICD}\left(A^{2 *(1)}\right)=0.1545, \operatorname{ICD}\left(A^{3 *(1)}\right)=0.1550$ and $\mathrm{GCD}=0.1428$

Since $\mathrm{GCD}=0.1428>\overline{\mathrm{GCD}}=0.1$, it is necessary to adjust the worst PFPR in the consensus. From the above operation results, we know $\operatorname{ICD}\left(A^{3 *(1)}\right)=\max _{1 \leq k \leq 3}\left\{\operatorname{ICD}\left(A^{k *(1)}\right)\right\}$. Then, the following $A^{3 *(1)}$ is adjusted by Eq. (35):

$$
A^{3 *(2)}=\left[\begin{array}{llll}
(\sqrt{2} / 2, \sqrt{2} / 2) & (0.7326,0.5648) & (0.7739,0.5128) & (0.7755,0.4538) \\
(0.5500,0.7500) & (\sqrt{2} / 2, \sqrt{2} / 2) & (0.6389,0.5382) & (0.6326,0.4967) \\
(0.4047,0.7972) & (0.4500,0.6500) & (\sqrt{2} / 2, \sqrt{2} / 2) & (0.5830,0.5685) \\
(0.4047,0.7515) & (0.4500,0.6128) & (0.4783,0.4509) & (\sqrt{2} / 2, \sqrt{2} / 2)
\end{array}\right],
$$

and let $A^{1 *(2)}=A^{1 *(1)}$ and $A^{2 *(2)}=A^{2 *(1)}$.

Next, the ICDs and GCD of the iterated PFPRs are calculated separately as follows: degree of group consensus.

\section{Stage 4: Alternative selection process}

Equation (30) is used to aggregate the PFPRs $A^{1 \Delta}, A^{2 \Delta}, \ldots, A^{m \Delta}$ into a comprehensive PFPR $A^{c \Delta}$. Accord-

ing to the ranking of the standardized weight vector calculated by the optimization model (M-4.2), the alternatives are sorted to select the best one $x^{\Delta}$

$\operatorname{ICD}\left(A^{1 *(2)}\right)=0.0809, \operatorname{ICD}\left(A^{2 *(2)}\right)=0.1085, \operatorname{ICD}\left(A^{3 *(2)}\right)=0.0710$ and $\mathrm{GCD}=0.0868$.

$$
\begin{aligned}
A^{c \Delta} & =\left(\left\langle a_{i j, \rho}^{c \Delta}, a_{i j, \sigma}^{c \Delta}\right\rangle\right)_{4 \times 4} \\
& =\left[\begin{array}{cccc}
(\sqrt{2} / 2, \sqrt{2} / 2) & (0.7299,0.5672) & (0.7784,0.5129) & (0.7762,0.4833) \\
(0.5687,0.7282) & (\sqrt{2} / 2, \sqrt{2} / 2) & (0.6321,0.5786) & (0.7004,0.5147) \\
(0.5708,0.7554) & (0.4893,0.6526) & (\sqrt{2} / 2, \sqrt{2} / 2) & (0.6515,0.5632) \\
(0.4557,0.7786) & (0.4778,0.6349) & (0.6144,0.5134) & (\sqrt{2} / 2, \sqrt{2} / 2)
\end{array}\right] .
\end{aligned}
$$

The matrix is applied to the model to obtain the normalized Pythagorean fuzzy weight vector

$\tilde{w}=\left(\tilde{w}_{1}, \tilde{w}_{2}, \tilde{w}_{3}, \tilde{w}_{4}\right)^{T}=(\langle 0.6424,0.6981\rangle,\langle 0.4609,0.8293\rangle,\langle 0.4037,0.9149\rangle,\langle 0.3345,0.9424\rangle)$, 
and its consistency index is: $C I\left(A^{\mathrm{c} \Delta}\right)=0.0196<\overline{C I}$.

Acknowledgements The work was supported by the National Natural Science Foundation of China (Nos. 91546108, 71901001, and 71701061), Natural Science Foundation of Anhui Province (No. 2008085QG333), Key Research Project of Humanities and Social Sciences in Colleges and Universities of Anhui Province (No. SK2020A0038), and the National Social Science Foundation of China (No. 16BJY132).

\section{Declarations}

Conflict of interest The authors declare no conflict of interest.

Open Access This article is licensed under a Creative Commons Attribution 4.0 International License, which permits use, sharing, adaptation, distribution and reproduction in any medium or format, as long as you give appropriate credit to the original author(s) and the source, provide a link to the Creative Commons licence, and indicate if changes were made. The images or other third party material in this article are included in the article's Creative Commons licence, unless indicated otherwise in a credit line to the material. If material is not included in the article's Creative Commons licence and your intended use is not permitted by statutory regulation or exceeds the permitted use, you will need to obtain permission directly from the copyright holder. To view a copy of this licence, visit http://creativecommons.org/licenses/by/4.0/.

\section{References}

1. Tanino T (1984) Fuzzy preference orderings in group decision making. Fuzzy Sets Syst 12:117-131

2. Milasi M, Puglisi A, Vitanza C (2019) On the study of the economic equilibrium problem through preference relations. J Math Anal Appl 477:153-162

3. Zhang C, Liao H, Luo L (2019) Additive consistency-based priority-generating method of q-rung orthopair fuzzy preference relation. Int J Intell Syst 34:2151-2176

4. Faizi S, Salabun W, Ullah S et al (2020) A new method to support decision-making in an uncertain environment based on normalized interval-valued triangular Fuzzy numbers and COMET technique. Symmetry 12:1-16

5. Faizi S, Rashid T, Sałabun W et al (2018) Decision making with uncertainty using hesitant fuzzy sets. Int J Fuzzy Syst 20:93-103

6. Zhou H, Wang J, Li X et al (2016) Intuitionistic hesitant linguistic sets and their application in multi-criteria decision making problems. Oper Res Int J 16:131-160

7. Wu P, Zhou L, Chen $\mathrm{H}$ et al (2019) Additive consistency of hesitant fuzzy linguistic preference relation with a new expansion principle for hesitant fuzzy linguistic term sets. IEEE Trans Fuzzy Syst 27:716-730

8. Liu J, Zheng Y, Jin F et al (2020) DEA cross-efficiency and fuzzy preference relation based on semi-disposability of undesirable outputs for environmental assessments. J Intell Fuzzy Syst 38:1-10

9. Zadeh LA (1965) Fuzzy sets. Inf Control 8:338-353

10. Orlovsky SA (1978) Decision-making with a fuzzy preference relation. Fuzzy Sets Syst 1:155-167

11. Xu ZS (2004) On compatibility of interval Fuzzy preference relations. Fuzzy Optim Decis Making 3:217-225

12. Al Salem AA, Awasthi A (2018) Investigating rank reversal in reciprocal fuzzy preference relation based on additive consistency: causes and solutions. Comput Ind Eng 115:573-581
13. Chang WJ, Fu C, Xu DL et al (2019) Triangular bounded consistency of fuzzy preference relations. Inf Sci 479:355-371

14. Xu YJ, Herrera F (2019) Visualizing and rectifying different inconsistencies for fuzzy reciprocal preference relations. Fuzzy Sets Syst 362:85-109

15. Xu ZS (2007) Intuitionistic preference relations and their application in group decision making. Inf Sci 177:2363-2379

16. Chen HP, Xu GQ (2019) Group decision making with incomplete intuitionistic fuzzy preference relations based on additive consistency. Comput Ind Eng 135:560-567

17. Liu JP, Song JM, Xu Q et al (2019) Group decision making based on DEA cross-efficiency with intuitionistic fuzzy preference relations. Fuzzy Optim Decis Making 18:345-370

18. Chu JF, Liu XW, Wang YM et al (2016) A group decision making model considering both the additive consistency and group consensus of intuitionistic fuzzy preference relations. Comput Ind Eng 101:227-242

19. Jin FF, Ni ZW, Chen HY et al (2016) Approaches to group decision making with intuitionistic fuzzy preference relations based on multiplicative consistency. Knowl Based Syst 97:48-59

20. Lin Y, Wang YM (2018) Group decision making with consistency of intuitionistic Fuzzy preference relations under uncertainty. IEEE/CAA J Autom Sin 5:741-748

21. Atanassov K (1986) Intuitionistic fuzzy sets. Fuzzy Sets Syst 20:87-96

22. Atanassov K (1989) More on intuitionistic fuzzy sets. Fuzzy Sets Syst 33:37-46

23. Yager RR, Abbasov AM (2013) Pythagorean membership grades, complex numbers, and decision making. Int J Intell Syst 28:436-452

24. Yager RR (2014) Pythagorean membership grades in multi-criteria decision making. IEEE Trans Fuzzy Syst 22:958-965

25. Garg H (2017) Generalized Pythagorean fuzzy geometric aggregation operators using Einstein t-norm and t-conorm for multicriteria decision-making process. Int J Intell Syst 32:597-630

26. Wu SJ, Wei GW (2016) Pythagorean fuzzy Hamacher aggregation operators and their application to multiple attribute decision making. Knowl Based Syst 97:24-39

27. Akram M, Peng XD, Alkenani A et al (2020) Prioritized weighted aggregation operators under complex pythagorean fuzzy information. J Intell Fuzzy Syst 39:4763-4783

28. Peng XD (2019) New similarity measure and distance measure for Pythagorean fuzzy set. Complex Intell Syst 5:101-111

29. Hussian Z, Yang MS (2019) Distance and similarity measures of Pythagorean fuzzy sets based on the Hausdorff metric with application to fuzzy TOPSIS. Int J Intell Syst 34:2633-2654

30. Ullah K, Mahmood T, Ali Z et al (2020) On some distance measures of complex Pythagorean fuzzy sets and their applications in pattern recognition. Complex Intell Syst 6:15-27

31. Zhang XL, Xu ZS (2015) Extension of TOPSIS to multiple criteria decision making with Pythagorean fuzzy sets. Int J Intell Syst 29:1061-1078

32. Akram M, Garg H, Zahid K (2020) Extensions of ELECTRE-I and TOPSIS methods for group decision-making under complex Pythagorean fuzzy environment. Iran J Fuzzy Syst 17:147-164

33. Peng XD, Selvachandran G (2019) Pythagorean fuzzy set: state of the art and future directions. Artif Intell Rev 52:1873-1927

34. Mandal P, Ranadive AS (2019) Pythagorean fuzzy preference relations and their applications in group decision-making systems. Int J Intell Syst 34:1700-1717

35. Yang Y, Qian GS, Ding H et al (2019) Pythagorean fuzzy preference relations and its application to group decision making. Control Decis 34:287-297

36. Zhang XL (2016) Multicriteria Pythagorean fuzzy decision analysis: A hierarchical QUALIFLEX approach with the closeness index-based ranking methods. Inf Sci 330:104-124

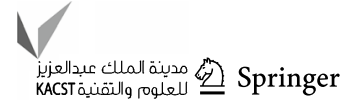


37. Zhang ZX, Hao WN, Yu XH et al (2019) Pythagorean fuzzy preference ranking organization method of enrichment evaluations. Int J Intell Syst 34:1416-1439

38. Zhang H (2019) Revisiting multiplicative consistency of interval fuzzy preference relation. Comput Ind Eng 132:325-332
39. He X, Liu WF, Chang J (2020) Multiplicative consistent Pythagorean fuzzy preference relation. Control Decis. https://doi.org/10. 13195/j.kzyjc.2019.0967

40. Peng XD, Yang Y (2015) Some results for Pythagorean fuzzy set. Int J Intell Syst 30:1133-1160 